\title{
Mode switching and standing-travelling waves in slug flow-induced vibration of catenary riser
}

\author{
Hongjun $\mathrm{Zhu}^{1,2 *}$, Yue $\mathrm{Gao}^{1}$, Narakorn Srinil ${ }^{3}$, Yan $\mathrm{Bao}^{4}$ \\ ${ }^{1}$ State Key Laboratory of Oil and Gas Reservoir Geology and Exploitation, Southwest Petroleum \\ University, Chengdu, China \\ ${ }^{2}$ State Key Laboratory of Coastal and Offshore Engineering, Dalian University of Technology, \\ China \\ ${ }^{3}$ Marine, Offshore \& Subsea Technology Group, School of Engineering, Newcastle University, \\ Newcastle Upon Tyne, United Kingdom \\ ${ }^{4}$ Department of Civil Engineering, Shanghai Jiao Tong University, Shanghai, China
}

\begin{abstract}
An experiment in a small-scale air-water test loop is performed to investigate the flow-induced vibration behaviors of a flexible catenary riser conveying slug flow over a wide range of the gas-toliquid flow rate ratios from 1.0 to 4.5. The clamped-clamped riser model has an inner diameter of 4 $\mathrm{mm}$ and an aspect ratio with the length over the inner diameter of about 360 . The non-intrusive highspeed cameras are employed to simultaneously capture the catenary riser dynamics and the internal unsteady slug flow characteristics. The space-time varying features of the slug flow-induced
\end{abstract} vibration are presented and discussed in terms of the response amplitudes, frequencies, modal components, and associated flow-induced forces. Slug flow properties including the translational velocities, lengths, fluctuation frequencies and pressure changes are reported. When increasing the ratio of the gas-to-liquid flow rates, experimental results highlight how a variation of the slug length and frequency contributes to a mode-switching phenomenon over time. This switching from the dominant first-mode frequency to the second-mode frequency leads to the associated transition from the standing wave to the travelling wave of the catenary riser response. The response amplitudes are amplified by the increased translational velocity and slug length, entailing the increased flow-

* Corresponding author. email: zhuhj@swpu.edu.cn (H. Zhu). 
induced momentum and gravitational forces, as the gas-to-liquid flow rate ratio is increased.

Consequently, higher vibration modes participate into the riser dynamics exhibiting a strong multimode competition.

Keywords: catenary riser; slug flow; flow-induced vibration; mode switching; standing wave; traveling wave.

\section{Nomenclature}

\begin{tabular}{|c|c|}
\hline$A_{\mathrm{c}}$ & Internal cross-sectional area of the riser \\
\hline$A_{\mathrm{X}} / D$ & Horizontal response amplitude per diameter \\
\hline$A_{\mathrm{Y}} / D$ & Out-of-plane response amplitude per diameter \\
\hline$A_{\mathrm{Z}} / D$ & Vertical response amplitude per diameter \\
\hline$A_{\mathrm{RMS}} / D$ & Root-mean-squared response amplitude per diameter \\
\hline$A_{\mathrm{X}, \mathrm{RMS}} / D$ & Root-mean-squared horizontal response amplitude per diameter \\
\hline$A_{\mathrm{Y}, \mathrm{RMS}} / D$ & Root-mean-squared out-of-plane response amplitude per diameter \\
\hline$A_{\mathrm{Z}, \mathrm{RMS}} / D$ & Root-mean-squared vertical response amplitude per diameter \\
\hline$A_{\mathrm{X}, \max } / D$ & Maximum horizontal response amplitude per diameter \\
\hline$A_{Z, \max } / D$ & Maximum vertical response amplitude per diameter \\
\hline$C_{\mathrm{s}}$ & Empirical coefficient for the liquid friction factor \\
\hline$D$ & Outer diameter of the riser \\
\hline$d$ & Internal diameter of the riser \\
\hline$E$ & Elastic modulus of the riser \\
\hline$F$ & Fluid force exerted on the riser by liquid slug \\
\hline$F_{X}$ & Horizontal component of fluid force exerted on the riser by liquid slug \\
\hline$F_{Z}$ & Vertical component of fluid force exerted on the riser by liquid slug \\
\hline$f$ & Response frequency \\
\hline$f_{\mathrm{x}}$ & Horizontal vibration frequency \\
\hline$f_{\mathrm{z}}$ & Vertical vibration frequency \\
\hline$f_{1 \mathrm{x}}, f_{2 \mathrm{x}}, f_{3 \mathrm{x}}$ & First three natural frequencies of flexible riser in $x$ direction \\
\hline$f_{1 \mathrm{y}}, f_{2 \mathrm{y}}, f_{3 \mathrm{y}}$ & First three natural frequencies of flexible riser in $y$ direction \\
\hline$f_{1 \mathrm{z}}, f_{2 \mathrm{z}}, f_{3 \mathrm{z}}$ & First three natural frequencies of flexible riser in $z$ direction \\
\hline$f_{\mathrm{d}}$ & Dominant vibration frequency \\
\hline$f_{\mathrm{m} 1}, f_{\mathrm{m} 2}, f_{\mathrm{m} 3}$ & Secondary frequencies ordered from the power spectral density (PSD) \\
\hline$f_{\mathrm{u}}$ & Recurrence frequency of slugs \\
\hline$f_{\mathrm{u}, \mathrm{min}}$ & Minimum recurrence frequency of slugs \\
\hline$f_{\mathrm{p}}$ & Fluctuation frequency of pressure at the riser base \\
\hline$f_{\mathrm{p} 1}-f_{\mathrm{p} 7}$ & Fluctuation frequencies of pressure at the riser base ranked by PSD \\
\hline$g$ & Gravitational acceleration \\
\hline$H$ & Vertical height of the riser \\
\hline$H_{\mathrm{s}}$ & Liquid holdup of the slug unit \\
\hline
\end{tabular}




\begin{tabular}{|c|c|}
\hline $\bar{H}_{\mathrm{s}}$ & Time-averaged liquid holdup of the slug unit \\
\hline$h_{\mathrm{s}}$ & Instantaneous liquid holdup in the whole riser \\
\hline$L_{\mathrm{u}} / D$ & Length of the slug unit per diameter \\
\hline$L_{\mathrm{s}} / D$ & Length of the liquid slug per diameter \\
\hline$L_{\mathrm{g}} / D$ & Length of the gas slug per diameter \\
\hline$L_{\mathrm{u}, \max } / D$ & Longest length of the slug unit per diameter \\
\hline$L_{\mathrm{s}, \max } / D$ & Longest length of the liquid slug per diameter \\
\hline$L_{\mathrm{g}, \max } / D$ & Longest length of the gas slug per diameter \\
\hline$l$ & Length of the riser \\
\hline$l_{0}$ & Horizontal span of the riser \\
\hline$l_{1}$ & Horizontal distance between pressure probes 1 and 2 \\
\hline$m_{\mathrm{s}}$ & Mass of liquid slug \\
\hline$n$ & Mode number \\
\hline$N_{m}$ & Number of markers along the riser \\
\hline$p$ & Fluid pressure \\
\hline$p_{1}$ & Monitored pressure by pressure probe 1 \\
\hline$p_{2}$ & Pressure at the riser base monitored by pressure probe 2 \\
\hline$p_{3}$ & Pressure at the riser top monitored by pressure probe 3 \\
\hline$p_{\mathrm{s} 1}$ & Local pressure at the bottom of the liquid slug \\
\hline$p_{\mathrm{s} 2}$ & Local pressure at the top of the liquid slug \\
\hline$p_{\text {RMS }}$ & Root-mean-squared pressure \\
\hline$Q_{\mathrm{G}}$ & Air volumetric flow rate \\
\hline$Q_{\mathrm{L}}$ & Water volumetric flow rate \\
\hline$Q_{\mathrm{G}} / Q_{\mathrm{L}}$ & Gas-to-liquid flow rate ratio \\
\hline$s / l$ & Curvilinear dimensionless coordinate along riser \\
\hline$t$ & Recorded time \\
\hline$t_{0}-t_{14}$ & Selected time instants \\
\hline$U_{\mathrm{t}}$ & Transitional velocity of slug unit \\
\hline$v_{\mathrm{m}}$ & Mixture velocity \\
\hline$v_{\mathrm{SG}}$ & Gas superficial velocity \\
\hline$v_{\mathrm{SL}}$ & Liquid superficial velocity \\
\hline$v_{\mathrm{m}} t / D$ & Dimensionless time \\
\hline$w$ & Modal weight \\
\hline$X / D$ & Horizontal vibration displacement per diameter \\
\hline$x$ & Horizontal coordinate \\
\hline$Y / D$ & Out-of-plane vibration displacement per diameter \\
\hline$Z / D$ & Vertical vibration displacement per diameter \\
\hline$z$ & Vertical coordinate \\
\hline$z / H$ & Dimensionless vertical location \\
\hline$\alpha_{1}$ & Local inclination angle at the liquid slug bottom \\
\hline$\alpha_{2}$ & Local inclination angle at the liquid slug top \\
\hline$\gamma$ & Empirical coefficient for the liquid friction factor \\
\hline$\rho_{\mathrm{r}}$ & Density of the riser \\
\hline$\rho_{\mathrm{s}}$ & Liquid density \\
\hline
\end{tabular}




$\begin{array}{ll}\zeta_{\text {in-plane }} & \text { In-plane damping ratio } \\ \zeta_{\text {out-of-plane }} & \text { Out-of-plane damping ratio } \\ \tau_{\mathrm{s}} & \text { Wall shear stress exerted by liquid slug } \\ \mu_{\mathrm{s}} & \text { Liquid kinematic viscosity } \\ \eta_{\mathrm{s}} & \text { Friction factor between the liquid slug and the riser wall }\end{array}$

\section{Introduction}

Slug flow is a typical and problematic flow regime in the transportation of oil and gas in offshore and subsea engineering, due to the time-space varying fraction and density of fluids and to the continuous fluctuation of pressure (Thaker and Banerjee, 2015; Al-Ruhaimani et al., 2018; Babakhani Dehkordi et al., 2019). The variation features of slug flows contribute to the unstable fluid loading on pipelines (Gourma and Verdin, 2020). The associated dynamic response is referred to as the slug flow-induced vibration (SIV), which influences the structural integrity and potentially causes the fatigue failure. been well documented. In contrast, understanding of the internal two-phase flow-induced vibration (FIV) is still limited as the associated literature involving both the two-phase flow and the fluidstructure interaction (FSI) mechanisms is scanty. Qrtiz-Vidal et al. (2017) have experimentally found that the mixture velocity, void fraction and flow regime of the two-phase, gas-liquid flow are the governing factors for FIV of a clamped-clamped straight horizontal pipe. An and Su (2015) numerically predicted that the pipe vibration amplitude increases with the gas volume fraction and the water flow rate. The experiments performed by Al-Hashimy et al. (2016) illustrated that the vibration displacement increases, while the predominant frequency decreases, when increasing the water superficial velocity. Other theoretical and experimental FIV studies were reported by Łuczko

51 and Czerwiński (2017), Bai et al. (2018), Wang et al. (2018) and Mohmmed et al. (2019). 
A curved pipe is subject to the reaction force exerted by the internal flow due to the change in the momentum flux direction. Bordalo and Morooka (2018) demonstrated that both catenary and lazy-wave risers can experience oscillations due to the internal liquid-gas mixtures. A severe vibration was observed by Riverin and Pettigrew (2007) in a U-shaped piping subject to the airwater flow. Pontaza and Menon (2011) numerically investigated the multiphase FIV of a subsea jumper and found that the internal FIV predominantly occurs within the jumper configuration plane. Jia (2012) investigated SIV responses of pipelines, jumpers, and risers by using the computational fluid dynamics approach. For a catenary riser, Chatjigeorgiou (2017) numerically predicted that the reduction of the slugging frequency leads to a stronger internal loading and hence larger displacements. Cabrera-Miranda and Paik (2019) employed the Euler-Bernoulli beam and steady slug flow models to investigate SIV of a steel lazy-wave riser. They concluded that the relatively small amplitudes presented in the upper part of the riser $(s / \geq 0.5$, where $s$ is the location measured from the riser base and $l$ is the total length) are due to the high slugging frequencies of the shortlength slugs. In contrast, the large-amplitude responses near the bottom end are caused by the energetic slug flow initiation near the riser inlet. Recently, Ma and Srinil (2020) modeled and investigated the effects of superficial gas-liquid velocities and slug unit characteristics (profile shape, length, velocity, frequency) on SIV responses of a long catenary riser subject to several resonance conditions. Nevertheless, it is still challenging to establish an appropriate model to formulate the transient characteristics of unsteady slug flows and to fully couple the space-time varying flow with the nonlinear dynamic response of the flexible pipe. A large-scale numerical simulation for a fully coupled FSI system is very time consuming and an experimental validation is required.

To date, a few experimental works have been carried out for SIV of curved flexible cylinders. 
Through a nonintrusive measurement, Zhu et al. (2018a) reported that a vigorous SIV response occurs when a consistent long liquid slug travels through the riser. The in-plane SIV is dominated by the fundamental mode in the gas-liquid flow rate ratio range of $0.5-1.827$. When increasing this flow rate ratio to 3.031 , a travelling wave characteristic of the fundamental mode dominates the inplane response (Zhu et al., 2019a). Three slugging flow regimes have been observed over a wide flow rate range. The response amplitude decreases and the dominant frequency increases as the flow regime evolves from the severe slugging to the relatively stable slug flow (Zhu et al. 2018b). Vieiro et al. (2019) conducted a small-scale experiment of a flexible lazy-wave riser transporting gas-liquid flow, showing a fundamental mode response. However, the detailed experimental results of internal flow characteristics were not sufficiently reported.

In this work, the aspect ratio of the catenary riser model is increased to about 360 and the range of the gas-liquid flow rate ratio is further broadened to be from 1.0 to 4.5 to involve higher excitation modes. The space-time varying responses and a mode switching phenomenon in SIV of the flexible catenary riser are discussed in terms of the response amplitudes, oscillation frequencies, flowinduced forces and the slug flow characteristics including the slug length, translational velocity, recurrence frequency and pressure fluctuation. The aim of this study is to improve understanding of the mode transition and competition mechanisms generated by SIV, and to provide more detailed experimental results of unstable slug flow-pipe response features for future model validations.

\section{Experimental Description}

\subsection{Two-phase flow facilities}

The experiments are carried out in the Offshore Oil and Gas Laboratory of the Southwest Petroleum University. Figure 1 depicts the air-water test loop employed to investigate SIV of a 
catenary riser. The main components consist of the water tank, air compressor, water pump, flowmeters, valves, and the T-type mixer. A compressed air is supplied from an electromagnetic air compressor (Model: ACO-016, power: $520 \mathrm{~W}$ ), which delivers air with the maximum discharge of 450 liter/minute at the standard room conditions (101325 $\mathrm{Pa}$ and $293.15 \mathrm{~K})$. The air flow rate is measured and regulated by a floater flowmeter (Model: LZB-4WB, accuracy of $\pm 2.5 \%$ ) and the associated stainless steel, ball-type valve, respectively. The water is pumped from a glass tank of volume $0.216 \mathrm{~m}^{3}$ by a submerged pump (Model: QDX1.5-16-0.37, power: $370 \mathrm{~W}$ ), which supplies water with the maximum discharge of $1.5 \mathrm{~m}^{3} /$ hour at the standard conditions. A liquid turbine-type flowmeter (Model: LWGY-B, accuracy of $\pm 0.5 \%$ ) and needle valve (Model: J13W-160P) are used to measure and regulate the water flow rate, respectively.

The air and water are mixed through the T-type mixer (a PVC-made tee junction with the same inner diameter of $4 \mathrm{~mm}$ as the upstream and downstream pipes). The two-phase mixture flows through a horizontal pipe with a length of $2.0 \mathrm{~m}$ ensuring a fully developed slug flow before the entrance to the test section. Two pressure transducers (Model: YPT1000-T02, accuracy of $\pm 0.1 \%$ ) are mounted on the horizontal pipe with one close to the entrance of the test section (pressure probe 2) and the other locating $1 \mathrm{~m}$ upstream (pressure probe 1). Another pressure transducer (pressure probe 3 ) is mounted at the outlet of the test section. These three transducers synchronously collect data with the sampling frequency of $100 \mathrm{~Hz}$, reporting the pressure changes over the catenary riser. Following the outlet, the air is discharged to the atmospheric condition via a funnel-shaped, airwater separator, whereas the water is continuously returned to the water tank by the gravity effect. Note that water in the storage tank is dyed black to clearly identify and distinguish the liquid slug from the elongated gas bubble for each slug unit. The present apparatus has been calibrated prior to 
the tests. Both air and water flow through the pipe system for about 20 minutes prior to the data acquisition. The pressure transducers are zeroed at the atmospheric pressure, recording the gauge pressure variations over time.

\subsection{Test section}

The test section is a fixed-fixed, flexible catenary riser made of the transparent silica gel with a length of $1.44 \mathrm{~m}$ and an internal diameter of $4 \mathrm{~mm}$, yielding an aspect ratio (length/diameter) of about 360 . This aspect ratio could provide some useful benchmarking results although the model diameter scale is very small when compared to actual diameters of subsea risers. The riser model is deployed as a catenary configuration in a vertical plane with the horizontal span $\left(l_{0}\right)$ and height $(H)$ of $1.029 \mathrm{~m}$ and $0.9 \mathrm{~m}$, respectively. The riser is only tensioned by its weight. The origin of the coordinate system is defined at the riser base as in Fig. 1. The density and elastic modulus of the flexible riser are $1041.8 \mathrm{~kg} / \mathrm{m}^{3}$ and $7.15 \mathrm{MPa}$, respectively. Table 1 summarizes the main parameters of the flexible riser model.

The non-intrusive measurement with high-speed cameras proposed in our previous studies (Zhu et al., 2016, 2019c) is employed in this work. Therefore, the riser model is evenly marked with 40 black rings with the width of $4 \mathrm{~mm}$ and the center-to-center distance of $36 \mathrm{~mm}$ along the span, providing the traceable markers for high-speed cameras. The markers $1^{\#}-40^{\#}$ are counted from the riser top to its base.

\subsection{Experimental matrix}

Eight gas-to-liquid flow rate ratios (GLRs) ranging from 1.0 to 4.5 with an increment of 0.5 are considered in this work, as listed in Table 2. The change in GLR is achieved by adjusting the opening of the gas control valve, while the water flow rate is kept unchanged. The gas superficial 
velocity varies from $0.508 \mathrm{~m} / \mathrm{s}$ to $2.288 \mathrm{~m} / \mathrm{s}$ while the water superficial velocity is fixed at 0.508 $\mathrm{m} / \mathrm{s}$. As GLR increases, the mixture velocity $\left(v_{\mathrm{m}}\right)$ increases from $1.016 \mathrm{~m} / \mathrm{s}$ to $2.796 \mathrm{~m} / \mathrm{s}$, within a common velocity range in practical operation, where $v_{\mathrm{m}}$ is defined as:

$$
v_{\mathrm{m}}=\frac{Q_{\mathrm{L}}+Q_{\mathrm{G}}}{A_{c}}
$$

where $Q_{\mathrm{L}}$ and $Q_{\mathrm{G}}$ are the water and air volumetric flow rates, respectively, and $A_{\mathrm{c}}$ is the internal cross-sectional area of the riser model.

\subsection{Non-intrusive measurement and processing}

Two high-speed cameras are used to capture the vibration displacements of 40 markers along the span as well as the slug flow characteristics. As shown in Fig. 1, one camera is pointed towards the curvature plane of the riser to record an in-plane response (xoz plane), and the other camera is placed above the riser to capture an out-of-plane response ( $y$ direction). The two cameras are triggered simultaneously with the resolution and sampling frequency of $2048 \times 1088$ pixels and 100 $\mathrm{Hz}$, respectively. The data acquisition period is $2 \mathrm{~min}$ for each GLR case, yielding a total of 12,000 images arrayed in time series from each camera. For each picture frame, one marker occupies 8-12 pixels, depending on a specific riser dynamic profile. The pixel calibration is conducted by corresponding the width of each marker in the recorded images to its actual size of $4 \mathrm{~mm}$. The vibration displacement of each marker is extracted by comparing its position from adjacent images, and the slug length is obtained by scaling the pixels occupied by the slug to the actual size. One can refer to our previous works for the image post-processing method and the precision examination test (Zhu et al., 2019a, 2019b).

The discretization study related to the number of markers is demonstrated in terms of the measured (discrete) dynamic displacement resolution affecting the numerically integrated (spatial) 
response profile. In comparison with an initial static shape, Fig. 2 illustrates the numerically obtained, maximum displacement profiles with the variation of the number of markers $\left(N_{m}\right)$ used in the camera recording, in the case of $Q_{\mathrm{G}} / Q_{\mathrm{L}}=3$. Correspondingly, Table 3 reports the maximum percent deviations of spatial displacement profiles in $x$ and $z$ directions when consecutively increasing $N_{m}$ from 10 to 45 . It can be seen that the percent deviations decrease with increasing $N_{m}$. The use of 40 discrete markers appears to provide a reasonable resolution with discrepancies being less than $3 \%$ and, therefore, it is applied to the present experimental SIV tests.

The original time series of displacements are obtained through the image post-processing. A band-pass filter $(0.3-8.0 \mathrm{~Hz})$ is applied to the response time histories to eliminate irrelevant bands of low and high frequencies, as exemplified in Fig. 3 in which the vertical response amplitude is defined as:

$$
A_{\mathrm{Z}}=Z-\bar{Z}
$$

where $Z$ and $\bar{Z}$ are the total and time-averaged vertical displacements, respectively.

\subsection{Decay test}

Free decay tests are conducted to identify natural frequencies and damping ratios of the catenary riser filled with air or water for both in-plane and out-of-plane directions. The free attenuation response is recorded after imposing an initial displacement on the riser. Figure 4 plots time histories of response displacements at the midspan (marker $21^{\#}$ ) and the corresponding frequency spectra. The fundamental frequencies of the flexible riser fully filled with air are found to be $2.32 \mathrm{~Hz}$ and $1.37 \mathrm{~Hz}$ in the in-plane and out-of-plane directions, with the associated damping ratios of 0.012 and 0.009 , respectively. As the system mass is increased when the riser is fully filled with water, the in-plane and out-of-plane fundamental frequencies are reduced to $2.20 \mathrm{~Hz}$ and 1.22 
$\mathrm{Hz}$, with the associated damping ratios of 0.011 and 0.007 , respectively. Results from the free decay tests are also summarized in Table 1.

Moreover, the modal analysis is conducted to identify the modal shapes in both in-plane and out-of-plane directions (Srinil et al. 2009). The first three modal shapes are depicted in Fig. 5. It is seen that the number of peaks and troughs of the modal shape in the in-plane direction is one more than that in the out-of-plane direction for the same mode, due to the in-plane catenary configuration.

\section{Results and Discussion}

\subsection{Mode switching at $Q_{\mathrm{G}} / Q_{\mathrm{L}}=3.0$}

\subsubsection{Response amplitude and frequency}

Fig. 6 displays the space-time varying responses of the catenary riser at $Q_{\mathrm{G}} / Q_{\mathrm{L}}=3.0$ with the associated root-mean-squared (RMS) amplitudes (dashed lines), where $s / l$ denotes the dimensionless arclength position over the riser length. It can be seen that the out-of-plane response $\left(A_{\mathrm{Y}} / D\right)$ is negligible when compared with the in-plane response $\left(A_{\mathrm{X}} / D\right.$ and $\left.A_{\mathrm{Z}} / D\right)$, consistent with the reported results in Riverin and Pettigrew (2007), Pontaza and Menon (2011), Al-Hashimy et al. (2016) and Zhu et al. (2018a, 2019a). Therefore, only the in-plane response is discussed in the following sections.

As shown in Fig. 6, the response amplitude in the horizontal direction $(x)$ is larger than that in the vertical direction $(z)$, especially in the upper part of the riser. This is attributed to the catenary deployment of the riser versus the inlet flow direction (Zhu et al., 2018a; Srinil et al., 2018). It is seen from the RMS amplitude distribution that the in-plane response is dominated by the fundamental mode. Nevertheless, the instantaneous profiles of the riser intermittently exhibit the $2^{\text {nd }}$ modal shape, implying a competition between the $1^{\text {st }}$ and $2^{\text {nd }}$ modes. Unlike a single-frequency 
vibration with an RMS amplitude of about $70.7 \%$, the peak RMS amplitudes in the $x$ and $z$ directions are about $52.4 \%$ and $57.6 \%$ of the corresponding maximum amplitudes, respectively, suggesting the coexistence of multiple modes. This multi-mode characteristic is also confirmed by a variable intersection of vibration envelops and a non-zero node of RMS amplitude along the span (Chaplin et al., 2005; Chaplin and King, 2018; Zhu et al., 2019c). The variation of instantaneous response mode versus a normalized time is plotted in Fig. 6 (b), demonstrating the mode order switching (between $n=1$ and 2) over time. The occurrence of the $2^{\text {nd }}$ mode is less than that of the fundamental one, illustrating the $1^{\text {st }}$ mode dominant response.

As shown in Fig. 7(a), a trough of the RMS amplitude profile appears at the marker $23^{\#}$ in the $z$ direction, $18 D$ below the midspan. The spatial asymmetric distribution of the RMS amplitude is due to the spanwise varying curvature of the catenary riser. The maximum RMS amplitude up to $2.30 \mathrm{D}$ occurs at the marker $14^{\#}$ in the $x$ direction, while it is about $1.54 \mathrm{D}$ at the marker $30^{\#}$ in the $z$ direction. The position of the marker $30^{\#}$ corresponds to the largest curvature, yielding a maximum centrifugal force and thereby the largest vertical response (Zhu et al., 2019b). The time series of the decomposed amplitudes at representative markers are displayed in Fig. 7(b). It is worth noting that the fundamental mode dominates the response at the two peaks of the RMS amplitude profile (markers $14^{\#}$ and $31^{\#}$ in the $x$ direction; markers $14^{\#}$ and $30^{\#}$ in the $z$ direction) while the second mode becomes the dominant one at the trough (marker $26^{\#}$ in the $x$ direction; $23^{\#}$ in the $z$ direction). This implies that a mode transition first occurs at the troughs where the vibration energy is relatively low. Similar values in the two decomposed amplitudes at the marker $3^{\#}$ also illustrate the $1^{\text {st }}$ versus $2^{\text {nd }}$ mode competition. Therefore, Fig. 7(b) signifies that the modal weight spatially varies along the riser span. 
Fig. 8 depicts the time-varying frequency analysis results of the in-plane vibration response.

At the locations around the peaks of SIV profile (see markers $12^{\#}, 14^{\#}, 17^{\#}$ and $30^{\#}$ in both $x$ and $z$ directions), the response energy mainly concentrates at the frequency of $2.19 \mathrm{~Hz}$ close to the fundamental natural frequency of the riser, confirming the predominant $1^{\text {st }}$ mode. In contrast, at the troughs of SIV profile (see marker $26^{\#}$ in the $x$ direction and $23^{\#}$ in the $z$ direction), the dominant frequency shifts to $3.63 \mathrm{~Hz}$ that falls between the second natural frequency $(3.46 \mathrm{~Hz})$ of the waterfilled riser and that $(3.83 \mathrm{~Hz})$ of the air-filled riser, indicating a resonance of the $2^{\text {nd }}$ mode. For the rest parts of the riser, both the low and high frequencies are present in the response, demonstrating the multi-mode oscillations with a modal frequency switching. The fluctuation of frequencies over time indicates an intermittent modal participation in the vibration process driven by the unsteady behavior of internal slug flow. Overall, the response energy at $2.19 \mathrm{~Hz}$ is greater than that at 3.63 $\mathrm{Hz}$ for the majority part of the riser in spite of the superposition of two modes. As a result, the fundamental mode dominates the response most of the time. In addition, the mode switching over time is observed to be synchronous in the two directions in spite of the spatial differences.

\subsubsection{Mode switching and slug flow hydrodynamics}

To further understand the mode switching, the slug flow characteristics, the time-space evolution of response profiles, and amplitudes at the three representative markers are presented in Fig. 9. In Fig. 9(a), a standing wave feature is observed in the spanwise response in the period I (75$77.5 \mathrm{~s})$ while this feature shifts to a travelling wave in the period II (79-84 s), suggesting the participation of the $2^{\text {nd }}$ mode and the co-existing modal oscillations as shown in Fig. 9(b). As reported in Chaplin et al. (2005) and Huera-Huarte et al. (2016), the standing wave behavior appears when the structure mainly responds in a mode with small contributions of adjacent modes, while 
the travelling wave occurs when the structure vibrates due to a combination of several modes (Srinil, 2011).

During the first period, the relatively long slug units pass through the riser with the maximum length of $159 \mathrm{D}$ and the associated liquid slug length up to $45 \mathrm{D}$, as shown in Fig. 9(c) for period I and 9(d) for period II. Moreover, only one complete slug unit is observed in the riser for each instantaneous snapshot. The presence of the longer liquid slug contributes to the greater vibration amplitudes, due to the effect of flow gravity and centrifugal forces moving along and acting on the riser. Since force sensors were not employed during the experiment, the dynamic forces exerted by each passing liquid slug or plug on the catenary riser may be calculated using the fluid momentum conservation as described in Seyed and Patel (1992). For a curved pipe segment with an internal diameter $d$, the flow-induced force components $\left(F_{\mathrm{X}}, F_{\mathrm{Z}}\right)$ exerted by the liquid slug in the horizontal $X$ and vertical $Z$ directions may be approximated through

$$
\begin{aligned}
& F_{\mathrm{X}}=\frac{\pi d^{2}}{4}\left[p_{\mathrm{s} 1} \cos \alpha_{1}-p_{\mathrm{s} 2} \cos \alpha_{2}+\rho_{\mathrm{s}}\left(U_{\mathrm{t}}^{2} \cos \alpha_{1}-U_{\mathrm{t}}^{2} \cos \alpha_{2}\right)\right] \\
& F_{\mathrm{Z}}=\frac{\pi d^{2}}{4}\left[p_{\mathrm{s} 1} \sin \alpha_{1}-p_{\mathrm{s} 2} \sin \alpha_{2}+\rho_{\mathrm{s}}\left(U_{\mathrm{t}}^{2} \sin \alpha_{1}-U_{\mathrm{t}}^{2} \sin \alpha_{2}-g L_{s}\right)\right]
\end{aligned}
$$

in which $\rho_{\mathrm{s}}$ is the liquid density, $g$ is the gravitational acceleration, $L_{s}$ is the liquid slug length, $U_{\mathrm{t}}$ is the estimated translational velocity, $\alpha_{1}$ and $\alpha_{2}$ are the local inclination angles measured from the $X$ axis, and $p_{\mathrm{s} 1}$ and $p_{\mathrm{s} 2}$ are the local pressure values, with the subscripts 1 and 2 denoting the bottom and top sections of the pipe segment, respectively. Note that the minor contributions of associated small gas bubbles (Ma and Srinil, 2020) have been neglected from Eqs. (3) and (4) which capture the effects of momentum flux, gravity and pressure change by the dominant liquid slug. Due to the gravity and friction effects, a pressure change occurs over the slug liquid length. By utilizing a slug 
hydrodynamic formulation of Taitel and Barnea (1990), $p_{\mathrm{s} 1}$ and $p_{\mathrm{s} 2}$ may be approximated through

$$
p_{s 1}-p_{s 2}=\rho_{\mathrm{s}} g L_{\mathrm{s}} \sin \left(\frac{\alpha_{1}+\alpha_{2}}{2}\right)+\frac{4 \tau_{\mathrm{s}}}{d} L_{\mathrm{s}}
$$

in which,

$$
\begin{aligned}
& \tau_{\mathrm{s}}=\eta_{\mathrm{s}}\left(\rho_{\mathrm{s}} U_{\mathrm{t}}\left|U_{\mathrm{t}}\right| / 2\right) \\
& \eta_{\mathrm{s}}=C_{\mathrm{s}}\left(U_{\mathrm{t}} d / \mu_{\mathrm{s}}\right)^{\gamma}
\end{aligned}
$$

where $\tau_{\mathrm{s}}$ is the wall shear stress exerted by the liquid slug, $\eta_{\mathrm{s}}$ is the friction factor between the liquid and the pipe wall, $\mu_{\mathrm{s}}$ is the liquid kinematic viscosity, and $C_{\mathrm{s}}$ and $\gamma$ are empirical coefficients. For the present turbulent slug flow with the Reynolds number in the range of about 4600 to 13400, being greater than $3000, C_{\mathrm{s}}=0.046$ and $\gamma=-0.2$ (Taitel and Dukler, 1976). As the pressure at the riser base $p_{2}$ is monitored by the pressure probe, the instantaneous pressure change along the riser length $\left(p_{\mathrm{s} 1}\right.$ and $\left.p_{\mathrm{s} 2}\right)$ can be approximated by successively applying Eq. (5) to every liquid slug portion. The calculated pressure at the riser top is then verified against the associated measured pressure at $p_{3}$. An error of less than $5 \%$ for the outlet pressure has been found in all time instants. Consequently, based on Eqs. (3) and (4), $F_{\mathrm{X}}$ and $F_{\mathrm{Z}}$ corresponding to the time instants in Fig. 9(c) and 9(d) are summarized in Table 4. It can be noticed that the fluid forces exerted by the longer liquid slugs are evidently greater than those induced by the shorter slugs. Both $F_{\mathrm{X}}$ and $F_{\mathrm{Z}}$ vary along the riser span as the slug flow moves upward.

Note that shorter slugs are transported through the riser during the second period in Fig. 9(d). The liquid slug lengths are mainly in the range of $16 D-22.2 D$, resulting in the smaller fluid forces exerted on the riser when compared to those imposed by the longer slugs during the first period, as shown in Table 4. Further, three or four liquid slugs simultaneously appear in the riser, imposing the fluid forces of moderate magnitudes on multiple segments of the riser. Consequently, the response 
amplitudes are relatively small during this period, as shown in Fig. 9(b). As two intersections are observed from the riser instantaneous profiles, the $2^{\text {nd }}$ modal response is captured. Therefore, the vibration amplitude is closely connected to the distribution of the liquid inventory along the riser depending on the slug length, slug frequency and holdup.

Table 4 also lists the slug flow parameters including the liquid slug length $\left(L_{\mathrm{s}}\right)$, translational velocity $\left(U_{\mathrm{t}}\right)$ and recurrence frequency $\left(f_{\mathrm{u}}\right)$ during the two considered periods in Fig. 9. It is seen that $U_{\mathrm{t}}$ values of slugs with different lengths are nearly the same due to the fixed inlet flow rate. The recurrence frequency of the $45 D$-length liquid slug is $2.65 \mathrm{~Hz}$, which is close to the first natural frequency and contributes to the $1^{\text {st }}$ mode dominant response. Similarly, the slug frequency associated with the liquid slug of length around $19 D$ is approximated as the $2^{\text {nd }}$ natural frequency, resulting in the $2^{\text {nd }}$ mode dominant response. In addition, some shorter slugs are conveyed in the riser with frequencies being higher than the $2^{\text {nd }}$ natural frequency due to the unsteady behavior of slug flow. This demonstrates the partial contribution from higher modes and the occurrence of mode competition or modal interaction (Srinil, 2010). It indicates that the SIV response is closely related to the slug frequency, which, in turn is associated with the slug length (Ma and Srinil, 2020).

Figure 10 displays variations of the monitored pressure at the riser base and outlet during the first period marked in Fig. 9. The pressure difference between the two monitored points $\left(p_{2}-p_{3}\right)$ represents the sum of the pressure loss and the static pressure formed by the liquid column in the riser. The static pressure is determined by the total liquid inventory accumulated in the riser. Consequently, the static pressure is less than $2 \mathrm{kPa}$ during $75.20-75.57 \mathrm{~s}$ and becomes greater than $3 \mathrm{kPa}$ during 75.73-75.83 s. Nevertheless, the static pressure contribution to the total pressure drop is small in comparison with the governing friction loss caused by the viscosity effect in a small- 
diameter pipe. In addition, the pressure fluctuation frequency coincides with the vibration frequency during this period, suggesting their interrelationship in spite of some phase differences. These will be further discussed in Section 3.1.3.

The transition from the standing wave to the travelling wave and the associated slug flow characteristic in the riser are displayed in Fig. 11. Occasionally, an intersection occurs between the instantaneous shape of the riser and its initial shape, illustrating the $1^{\text {st }}$ mode response. The presence of two intersections indicates the $2^{\text {nd }}$ mode becoming the predominant one. It demonstrates a strong competition and switching between the $1^{\text {st }}$ and $2^{\text {nd }}$ modes during the transition process. Moreover, the liquid slugs with lengths from $19.8 D$ to $31.2 D$ are transmitted through the riser with the corresponding slug frequency changing from $2.95 \mathrm{~Hz}$ to $3.87 \mathrm{~Hz}$, which is between the $1^{\text {st }}$ and the $2^{\text {nd }}$ natural frequencies. It indicates that the transition from the standing wave to the travelling wave is associated with the mode switching from the $1^{\text {st }}$ to the $2^{\text {nd }}$ and the shifted dominant frequency.

\subsubsection{Relationship among vibration, pressure fluctuation and slug flow frequencies}

Fig. 12 compares the related frequencies including the vibration frequencies $\left(f_{\mathrm{d}}\right.$ is the dominant one, whereas $f_{\mathrm{m} 1}, f_{\mathrm{m} 2}$ and $f_{\mathrm{m} 3}$ are secondary frequencies being ordered in terms of the power spectral densities), the recurrence frequencies of slugs $\left(f_{\mathrm{u}}\right)$ and the fluctuation frequencies of pressure at the riser base $\left(f_{\mathrm{p} 1}-f_{\mathrm{p} 7}\right.$ being ranked by the power spectral densities for which $f_{\mathrm{p} 1}$ is the most pronounced one). It is remarked that the pressure at the riser base (the test section inlet) fluctuates during a long period of $100 \mathrm{~s}$ as the riser conveys liquid slugs with lengths in a wide range of $13.8 D-48 D$. The longer the liquid slug, the smaller the recurrence frequency. The recurrence frequencies for the $48 D$ length and $45 \mathrm{D}$-length liquid slugs are $1.98 \mathrm{~Hz}$ and $2.65 \mathrm{~Hz}$, respectively. The fluctuation frequencies of the pressure are generally in consistent with the recurrence frequencies of liquid slugs. 
The majority of the riser part oscillates at the dominant frequency of $2.19 \mathrm{~Hz}$, except for the part around the trough of SIV profile (see Fig. 7) where the dominant frequency of $3.63 \mathrm{~Hz}$ is present. The two vibration frequencies coincide with the recurrence frequencies of liquid slugs with lengths of $45 D-48 D$ and $18.6 D-31 D$, respectively. It implies the $1^{\text {st }}$ mode dominant response which is mainly induced by the liquid slugs with the length of $45 D-48 D$, while the $2^{\text {nd }}$ mode response emerges by shortening the liquid slug to be less than $31 D$. The occurrence of other higher vibration frequencies is mainly attributed to the shorter slugs passing through the riser with higher frequencies. The shortest liquid slug of length $13.8 D$ possesses the highest recurrence frequency $f_{\mathrm{u}}=9.03 \mathrm{~Hz}$, which is close to the highest frequency of the pressure $f_{\mathrm{p} 7}=9.23 \mathrm{~Hz}$. Therefore, the pressure fluctuation and the vibration response are closely related to the recurrence frequency of slugs.

\subsection{Effect of gas-liquid flow rate ratio}

\subsubsection{Vibration response}

The spanwise evolutions of RMS amplitudes at different GLRs are compared in Fig. 13. As GLR increases from 1.0 to 4.5 , the vibration amplitude increases gradually, consistent with the reported results in An and Su (2015), Ma et al. (2020) and Zhu et al. (2019a). The RMS amplitudes achieve the maximum values up to $3.31 D$ and $2.01 D$ in the $x$ and $z$ directions, respectively, at $Q_{\mathrm{G}} / Q_{\mathrm{L}}=4.5$. Nevertheless, the growth rate of vibration amplitude becomes slower as $Q_{\mathrm{G}} / Q_{\mathrm{L}}$ increases from 3.5 to 4.5 , which is possibly related to the variation of modal weights. The profiles of RMS amplitudes illustrate that the in-plane responses in all the considered cases are dominated by the fundamental mode, due mainly to the considered low GLR and riser length.

Nevertheless, the contribution of higher modes increases with GLR. As shown in Fig. 14, the instantaneous shapes of the riser mainly display the $1^{\text {st }}$ mode at $Q_{\mathrm{G}} / Q_{\mathrm{L}}=1.0$ and 2.0 , and the $2^{\text {nd }}$ mode 
is occasionally observed at $Q_{\mathrm{G}} / Q_{\mathrm{L}}=2.0$. The occurrence frequency of the $2^{\text {nd }}$ modal shape is significantly increased as $Q_{\mathrm{G}} / Q_{\mathrm{L}}$ increases from 2.0 to 4.5 . At $Q_{\mathrm{G}} / Q_{\mathrm{L}}=4.5$, the $3^{\text {rd }}$ modal participation is also sometimes observed, indicating the higher-mode competition. Moreover, the deviations of nodes in both horizontal and vertical directions become greater with increasing GLR, signifying the growing contribution of higher modes.

Figure 15 compares the frequency spectra at different GLRs, where the dot-dash lines represent the first and second natural frequencies of the water-filled riser $\left(f_{1 \mathrm{z}}=2.2 \mathrm{~Hz}\right.$ and $\left.f_{2 \mathrm{z}}=3.46 \mathrm{~Hz}\right)$, and the dashed line outlines the predominant frequency along the riser span. As the fundamental mode dominates the in-plane response in all eight cases, the majority part of the catenary riser oscillates in a frequency very close to the first natural frequency. Nevertheless, the dominant frequency shifts to the second natural frequency at a position corresponding to the trough of vibration profile (marker $\left.23^{\#}\right)$. Furthermore, the region dominated by the $2^{\text {nd }}$ mode is gradually extended from the trough to both sides as GLR increases. It is extended to $15 \%$ of the riser at $Q_{\mathrm{G}} / Q_{\mathrm{L}}=4.5$. It demonstrates that the mode transition originates from the trough.

Figure 16 plots modal weights of the first three modes versus GLR. The modal weight of the $1^{\text {st }}$ mode decreases from $91.14 \%$ to $57.2 \%$ as GLR increases from 1.0 to 4.5 , while contributions of both $2^{\text {nd }}$ and $3^{\text {rd }}$ modes increase gradually. The maximum $2^{\text {nd }}$ and $3^{\text {rd }}$ modal weights reach $32 \%$ and $10.8 \%$, respectively, at $Q_{\mathrm{G}} / Q_{\mathrm{L}}=4.5$. Nevertheless, the growth rate is relatively slow (about half of the corresponding growth rate with increasing $Q_{\mathrm{G}} / Q_{\mathrm{L}}$ from 1.0 to 3.5 ) with increasing $Q_{\mathrm{G}} / Q_{\mathrm{L}}$ from 3.5 to 4.5 , coinciding with the growing vibration amplitude (see Fig. 13).

As shown in Fig. 17, at $Q_{\mathrm{G}} / Q_{\mathrm{L}}=1.0$ and 1.5, the response presents the standing wave characteristic along the riser span with the vibration frequency predominant at $2.21 \mathrm{~Hz}$. Although 
the standing wave is pronounced in the spanwise response, the travelling wave emerges at $Q_{\mathrm{G}} / Q_{\mathrm{L}}=$ 2.0, which is associated with the growing modal weights of higher modes and the participation of higher frequencies $(3.73 \mathrm{~Hz}$ and $5.6 \mathrm{~Hz})$. As GLR is further increased from 2.0 to 4.5 , the occurrence frequency of travelling wave grows gradually, accompanying with a more frequent switching between standing and travelling waves. Additionally, more frequencies participate into the response, illustrating the strengthened mode competition. As seen in the right column of Fig. 17, the response energy is gradually transferred from the fundamental frequency to the higher ones, with increasing GLR, justifying the mode transition mechanism.

\subsubsection{Slug flow characteristics and the relationship of frequencies}

The maximum lengths of slugs and the time-averaged liquid holdup $\left(\bar{H}_{s}\right)$ in the riser are compared in Fig. 18. The increased gas entering the test loop results in the significant increase of the elongated gas length and consequently the increased slug unit length. The maximum slug unit length reaches $351 D$ at $Q_{\mathrm{G}} / Q_{\mathrm{L}}=4.5$, about ten times of that at $Q_{\mathrm{G}} / Q_{\mathrm{L}}=1.0$. It is noted that the liquid slug length is also increased with increasing GLR, but the growth rate is much smaller than that of the gas length. This leads to the decreased liquid holdup in the riser. At $Q_{\mathrm{G}} / Q_{\mathrm{L}}=1.0$, the maximum liquid slug length is $24.33 D$, while it is increased to $75 D$ at $Q_{\mathrm{G}} / Q_{\mathrm{L}}=4.5$. In the upstream horizontal section, the accelerating gas prompts the thinning of the liquid film below the long gas bubble and consequently the addition of liquid into the liquid slugs. As a result, the developed slugs in the riser possess longer liquid columns. This phenomenon coincides with the reported results in Paglianti et al (1996) and Al-Kayiem et al. (2017). The translational velocity of slugs increases with GLR because of the growing flow rate. When compared with the translational velocity at $Q_{\mathrm{G}} / Q_{\mathrm{L}}=1.0$, the mean $U_{\mathrm{t}}$ is almost tripled at $Q_{\mathrm{G}} / Q_{\mathrm{L}}=4.5$. The higher slug translational velocity at the higher 
$Q_{\mathrm{G}} / Q_{\mathrm{L}}$ results in the greater flow-induced momentum forces $F_{\mathrm{X}}$ and $F_{\mathrm{Z}}\left(U_{t}^{2}\right.$ terms in Eqs. (3) and (4)) and, hence, the greater oscillation amplitudes. In addition, the corresponding slug unit length is increased by up to nine times at $Q_{\mathrm{G}} / Q_{\mathrm{L}}=4.5$ when compared to that at $Q_{\mathrm{G}} / Q_{\mathrm{L}}=1.0$. The longer and heavier liquid slug length generates greater and more localized gravitational force $F_{Z}$ (Eq. 4) which, in turn, further affects the riser configuration and vibration response (Ma and Srinil 2020). With the less-frequent longer slugs, the recurrence slug frequency is therefore reduced with the increased gas-liquid ratio, as listed in Table 5. At a result, the larger amplitude responses are observed at a lower slug frequency, in agreement with numerical prediction results in Chatjigeorgiou (2017). It is worth pointing out that the longest slug unit and the longest liquid slug do not necessarily occur at the same time, as shown in Table 6.

Fig. 18(b) also compares the distribution of the liquid inventory along the riser at different GLRs. The smaller the GLR ratio, the greater the liquid slugs presenting in the riser. There are nineteen liquid slugs that simultaneously occur when $Q_{\mathrm{G}} / Q_{\mathrm{L}}=1.0$. Nevertheless, each of them is relatively short around $6.0 D$, excluding the longest one that reaches $24 D$, as listed in Table 7 . The location of each liquid slug and the associated force imposed on the riser are also listed in this table. It is clearly seen that forces exerted by the shorter slugs are relatively smaller than those exerted by the longest ones. Thus, the longest liquid slug is the main contributor to the vibration displacement. With the increase of $Q_{\mathrm{G}} / Q_{\mathrm{L}}$ from 1.0 to 2.5 , the number of liquid slugs gradually decrease from 19 to 5, while their lengths are increased. When $Q_{\mathrm{G}} / Q_{\mathrm{L}} \geq 3.0$, only one or two liquid slugs are observed in the riser. The imposed forces become greater with the elongation of liquid slugs, resulting in the vibration enhancement. Consequently, the response is closely related to the behavior of the longest liquid slug when $Q_{\mathrm{G}} / Q_{\mathrm{L}} \geq 3$. 
Fig. 19 compares three interrelated response frequencies at different GLRs. It is interesting to notice that the dominant vibration frequency $\left(f_{\mathrm{d}}\right)$ is close to the most pronounced frequency of the pressure fluctuation $\left(f_{\mathrm{p} 1}\right)$, signifying a resonance between the vibration response and the pressure fluctuation. Both of the two resonant frequencies are associated with the slug flow characteristics. When $Q_{\mathrm{G}} / Q_{\mathrm{L}}<2.5, f_{\mathrm{d}}$ coincides well with $f_{\mathrm{p} 1}$, while the two frequencies do not exactly match when $Q_{\mathrm{G}} / Q_{\mathrm{L}} \geq 2.5$. Short slugs are transmitted through the riser with high frequencies when $Q_{\mathrm{G}} / Q_{\mathrm{L}}<2.5$.

Consequently, multiple liquid slugs appear in the riser at the same time (see Fig. 18 (b)), dispersing the fluid forces acting on the riser and hence leading to small response amplitudes. Therefore, the catenary riser response is dominated by the unsteady behavior of the slug flow that is governed and characterized by the pressure fluctuation when $Q_{\mathrm{G}} / Q_{\mathrm{L}}<2.5$. In contrast, the number of slugs simultaneously occurring in the riser are reduced at the high GLRs, resulting in the concentration riser base where it is basically free from the fluid-riser interaction effect. 
probability density function (PDF). The pressure decreases along the flow direction, i.e. $p_{1}>p_{2}>$ $p_{3}$, due to the head loss. The pressure difference between the riser base and top $\left(p_{2}-p_{3}\right)$ represents a pressure drop, which is gradually reduced as GLR is increased. The main reason is that the summative length of liquid slugs appearing in the riser becomes shorter, on average over time, with the increased $Q_{\mathrm{G}} / Q_{\mathrm{L}}$, giving rise to a lower liquid holdup and liquid column pressure. It is seen that the pressure band becomes wider as $Q_{\mathrm{G}} / Q_{\mathrm{L}}$ grows, which is associated with the broader range of the liquid slug length, as listed in Table 5. Additionally, with increasing GLR, the occurrences of slugs with more different lengths amplify the pressure fluctuation and consequently the increase of RMS pressure, as illustrated in Fig. 20 (b).

\section{Conclusions}

Slug flow-induced vibration (SIV) of a clamped-clamped flexible catenary riser transporting an air-water flow with variable gas-to-liquid flow rate ratios $Q_{\mathrm{G}} / Q_{\mathrm{L}}$ has been experimentally investigated to demonstrate and characterize the mode transition and competition mechanisms generated by SIV. Key findings are summarized as follows.

(1) In-plane responses are dominated by the fundamental mode in all the considered $Q_{\mathrm{G}} / Q_{\mathrm{L}}$ cases. An intermittent participation of higher modes leads to a distinct mode switching over time, closely related to the variation of the slug length and frequency. The predominant fundamental mode response is mainly induced by the liquid slugs with lengths of $45 D-48 D$ and by the recurrence frequencies near the fundamental natural frequency. The second-mode dominant response is primarily attributed to the occurrence of shorter liquid slugs with lengths of $18.6 D-31 D$ and by the associated fluctuation frequencies close to the second natural frequency. Further, a switching from the dominant first mode to the second mode is associated with a transition from the standing wave 
to the travelling wave, together with an interexchange between their associated natural frequencies. (2) As $Q_{\mathrm{G}} / Q_{\mathrm{L}}$ is less than 2.5 , multiple short slugs are present in the catenary riser, leading to the small-amplitude SIV response that is dominated by the unsteady behavior of the transient slug flow. In contrast, apart from the pronounced frequency of the pressure fluctuation, the response at $Q_{\mathrm{G}} / Q_{\mathrm{L}} \geqslant 2.5$ is closely related to the occurrence of long slugs possessing higher translational velocities, imposing greater and more concentrated flow-induced momentum and gravitational forces on the catenary riser exhibiting larger vibration amplitudes.

(3) A mode transition occurs temporally and spatially along the riser span. It first occurs at the trough of SIV profile and expands from the trough to both ends as $Q_{\mathrm{G}} / Q_{\mathrm{L}}$ increases. Due to a resonance between the slug frequencies and the riser natural frequencies, the modal weights of higher modes grow gradually, resulting in the pronounced multi-mode characteristics and appearance of travelling wave. In addition, both the slug length and translation velocity are enlarged with the increased $Q_{\mathrm{G}} / Q_{\mathrm{L}}$, giving rise to the enhanced SIV and multi-mode responses.

Further experimental investigation is required for larger-scale flexible risers experiencing the higher-order modal responses and resonances to improve understanding of slug-riser interactions of practical relevance.

\section{Acknowledgements}

The research work was supported by National Natural Science Foundation of China (Nos. 51979238 and 11772193) and the Open Research Fund of State Key Laboratory of Coastal and Offshore Engineering, Dalian University of Technology (No. LP1930). The authors appreciate the support from the Offshore Oil and Gas Laboratory at Southwest Petroleum University as well as the UK-

China International Exchanges Grant (IEC/NSFC/181370) from the Royal Society in the UK for 
491

supporting this research collaboration. N Srinil is also grateful for the funding support from EPSRC, part of UK Research and Innovation, through the MUFFINS project (EP/P033148/1) for methodology and theoretical discussion.

\section{References}

Al-Hashimy, Z.I., Al-Kayiem, H.H., Time, R.W., 2016. Experimental investigation on the vibration induced by slug flow in horizontal pipe. ARPN J. Eng. Appl. Sci. 11, 12134-12139.

Al-Kayiem, H.H., Mohmmed, A.O., Al-Hashimy, Z.I., Time, R.W., 2017. Statistical assessment of experimental observation on the slug body length and slug translational velocity in a horizontal pipe. Int. J. Heat Mass Tran. 105, 252-260.

Al-Ruhaimani, F., Pereyra, E., Sarica, C., Al-Safran, E., Chung, S., Torres, C., 2018. A study on the effect of high liquid viscosity on slug flow characteristics in upward vertical flow. J. Petrol. Sci. Eng. 161, 128-146.

An, C., Su, J., 2015. Dynamic behavior of pipes conveying gas-liquid two-phase flow. Nucl. Eng. Des. 292, 204-212.

Babakhani Dehkordi, P., Colombo, L.P.M., Mohammadian, E., Arnone, D., Azdarpou, A., Sotgia, G., 2019. Study of viscous oil-water-gas slug flow in a horizontal pipe. J. Petrol. Sci. Eng. 178, $1-13$.

Bai, Y., Xie, W., Gao, X., Xu, W., 2018. Dynamic analysis of a cantilevered pipe conveying fluid with density variation. J. Fluids Struct. 81, 638-655.

Bordalo, S.N., Morooka, C.K., 2018. Slug flow induced oscillations on subsea petroleum pipelines. J. Petrol. Sci. Eng. 165, 535-549.

Cabrera-Miranda, J.M., Paik, J.K., 2019. Two-phase flow induced vibrations in a marine riser conveying a fluid with rectangular pulse train mass. Ocean Eng. 174, 71-83. 
514 Chatjigeorgiou, I.K., 2017. Hydroelastic response of marine risers subjected to internal slug-flow. $515 \quad$ Appl. Ocean Res. 62, 1-17.

516 Chaplin, J.R., Bearman, P.W., Huera Huarte, F.J., Pattenden, R.J., 2005. Laboratory measurements of vortex-induced vibrations of a vertical tension riser in a stepped current. J. Fluids Struct. 21, Gourma, M., Verdin, P.G., 2020. Nature and magnitude of operating forces in a horizontal bend conveying gas-liquid slug flows. J. Petrol. Sci. Eng. 190, 107062.

Huera-Huarte, F.J., Bangash, Z.A., Gonzalez, L.M., 2016. Multi-mode vortex and wake-induced vibrations of a flexible cylinder in tandem arrangement. J. Fluids Struct. 66, 571-588.

Jia, D., 2012. Slug flow induced vibration in a pipeline span, a jumper, and a riser section. In: 2012 Offshore Technology Conference, Houston, USA.

Łuczko, J., Czerwiński, A., 2017. Nonlinear three-dimensional dynamics of flexible pipes conveying fluids. J. Fluids Struct. 70, 235-260.

Ma, B., Srinil, N., Zhu, H., Gao, Y., 2020. Experiment on the effect of superficial gas-liquid velocities on slug flow-induced vibration in an inclined sagged riser. Proceedings of the ASME 39th International Conference on Ocean, Offshore \& Arctic Engineering, OMAE2020-18034.

Ma, B., Srinil, N., 2020. Planar dynamics of inclined curved flexible riser carrying slug liquid-gas flows. J. Fluids Struct. 94, 102911.

Mohmmed, A.O., Al-Kayiem, H.H., Nasif, M.S., Time, R.W., 2019. Effect of slug flow frequency on the mechanical stress behavior of pipelines. Int. J. Pres. Ves. Pip. 172, 1-9. 
Ortiz-Vidal, L.E., Mureithi, N.W., Rodriguez, O.M.H., 2017. Vibration response of a pipe subjected to two-phase flow: Analytical formulations and experiments. Nucl. Eng. Des. 313, 214-224.

Paglianti, A., Giona, M., Soldati, A., 1996. Characterization of subregimes in two-phase slug flow. Int. J. Multiphase Flow 22, 783-796.

Pontaza, J.P., Menon. R.G., 2011. Flow-induced vibrations of subsea jumpers due to internal multiphase flow. In: $30^{\text {th }}$ International Conference on Ocean, Offshore and Arctic Engineering, Rotterdam, The Netherlands.

Riverin, J.L., Pettigrew, M.J., 2007. Vibration excitation forces due to two-phase flow in piping elements. J. Pres. Ves. Technol. 129, 7-13.

Seyed, F.B., Patel, M.H., 1992. Mathematics of flexible risers including pressure and internal flow effects. Mar. Structures 5, 121-150.

Srinil, N., Wiercigroch, M., O'Brien, P., 2009. Reduced-order modelling of vortex-induced vibration of catenary riser. Ocean Eng. 36, 1404-1414.

Srinil, N., 2010. Multi-mode interactions in vortex-induced vibrations of flexible curved/straight structures with geometric nonlinearities. J. Fluids Struct. 26, 1098-1122.

Srinil, N., 2011. Analysis and prediction of vortex-induced vibrations of variable-tension vertical risers in linearly sheared currents. Appl. Ocean Res. 33, 41-53.

Srinil, N., Ma, B., Zhang, L., 2018. Experimental investigation on in-plane/out-of-plane vortexinduced vibrations of curved cylinder in parallel and perpendicular flows. J. Sound Vib. 421, $275-299$.

Taitel, Y., Barnea, D., 1990. Two-phase slug flow. Advances in heat transfer. Elsevier, 1990.

Taitel, Y., Dukler, A.E., 1976. A model for predicting flow regime transitions in horizontal and near 
Thaker, J., Banerjee, J., 2015. Characterization of two-phase slug flow sub-regimes using flow visualization. J. Petrol. Sci. Eng. 135, 561-576.

Vieiro, J.J., Akhiiartdinov, A., Sævik, S., Larsen, C.M., Nydal, O.J. 2019. Two-way coupled fluidstructure interaction of gas-liquid slug flow in a flexible riser: small-scale experiments and simulations. Multiphase Sci. Technol. 31, 27-43.

Wang, L., Yang, Y., Li, Y., Wang, Y., 2018. Dynamic behaviours of horizontal gas-liquid pipes subjected to hydrodynamic slug flow: Modelling and experiments. Int. J. Pres. Ves. Pip. 161, $50-57$.

Zhu, H., Lin, P., Yao, J., 2016. An experimental investigation of vortex-induced vibration of a curved flexible pipe in shear flows. Ocean Eng. 121, 62-75.

Zhu, H., Gao, Y., Zhao, H., 2018a. Experimental investigation on the flow-induced vibration of a free-hanging flexible riser by internal unstable hydrodynamic slug flow. Ocean Eng. 164, 488507.

Zhu, H., Zhao, H., Gao, Y., 2018b. Experimental investigation of vibration response of a freehanging flexible riser induced by internal gas-liquid slug flow. China Ocean Eng. 32, 633-645.

Zhu, H., Gao, Y., Zhao, H., 2019a. Experimental investigation of slug flow-induced vibration of a flexible riser. Ocean Eng. 189, 106370.

Zhu, H., Gao, Y., Zhao, H., 2019b. Coupling vibration response of a curved flexible riser under the combination of internal slug flow and external shear current. J. Fluids Struct. 91, 102724.

Zhu, H., Lin, P., Gao, Y., 2019c. Vortex-induced vibration and mode transition of a curved flexible free-hanging cylinder in exponential shear flows. J. Fluids Struct. 84, 56-76. 
Table 1. Main parameters of the flexible catenary riser model.

\begin{tabular}{|c|c|c|}
\hline Parameters & Values & Units \\
\hline Length, $l$ & 1.440 & $\mathrm{~m}$ \\
\hline Internal diameter, $d$ & 0.004 & $\mathrm{~m}$ \\
\hline Outer diameter, $D$ & 0.006 & $\mathrm{~m}$ \\
\hline Vertical height, $H$ & 0.900 & $\mathrm{~m}$ \\
\hline Elastic modulus, $E$ & 7.15 & MPa \\
\hline Density of the riser, $\rho_{\mathrm{r}}$ & 1041.8 & $\mathrm{~kg} / \mathrm{m}^{3}$ \\
\hline The first-order natural frequency (water filled), $f_{1 \mathrm{x}}, f_{1 \mathrm{y}}, f_{1 \mathrm{z}}$ & $2.20,1.22,2.20$ & $\mathrm{~Hz}$ \\
\hline The second-order natural frequency (water filled), $f_{2 \mathrm{x}}, f_{2 \mathrm{y}}, f_{2 \mathrm{z}}$ & $3.46,2.55,3.46$ & $\mathrm{~Hz}$ \\
\hline The third-order natural frequency (water filled), $f_{3 x}, f_{3 y}, f_{3 z}$ & $5.45,4.13,5.45$ & $\mathrm{~Hz}$ \\
\hline The first-order natural frequency (air filled), $f_{1 \mathrm{x}}, f_{1 \mathrm{y}}, f_{1 \mathrm{z}}$ & $2.32,1.37,2.32$ & $\mathrm{~Hz}$ \\
\hline The second-order natural frequency (air filled), $f_{2 \mathrm{x}}, f_{2 \mathrm{y}}, f_{2 \mathrm{z}}$ & $3.83,2.71,3.83$ & $\mathrm{~Hz}$ \\
\hline The third-order natural frequency (air filled), $f_{3 x}, f_{3 y}, f_{3 z}$ & $5.66,4.30,5.66$ & $\mathrm{~Hz}$ \\
\hline Damping ratio (water filled), $\zeta_{\text {in-plane }}$ & 0.011 & Dimensionless \\
\hline Damping ratio (air filled), $\zeta_{\text {in-plane }}$ & 0.012 & Dimensionless \\
\hline Damping ratio (water filled), $\zeta_{\text {out-of-plane }}$ & 0.007 & Dimensionless \\
\hline Damping ratio (air filled), $\zeta_{\text {out-of-plane }}$ & 0.009 & Dimensionless \\
\hline
\end{tabular}


Table 2. Experimental case studies.

\begin{tabular}{ccccc}
\hline Cases & $v_{\mathrm{SG}}(\mathrm{m} / \mathrm{s})$ & $v_{\mathrm{SL}}(\mathrm{m} / \mathrm{s})$ & $v_{\mathrm{m}}(\mathrm{m} / \mathrm{s})$ & $Q_{\mathrm{G}} / Q_{\mathrm{L}}$ \\
\hline 1 & 0.508 & 0.508 & 1.016 & 1.0 \\
2 & 0.763 & 0.508 & 1.271 & 1.5 \\
3 & 1.017 & 0.508 & 1.525 & 2.0 \\
4 & 1.271 & 0.508 & 1.779 & 2.5 \\
5 & 1.525 & 0.508 & 2.033 & 3.0 \\
6 & 1.779 & 0.508 & 2.287 & 3.5 \\
7 & 2.033 & 0.508 & 2.541 & 4.0 \\
8 & 2.288 & 0.508 & 2.796 & 4.5 \\
\hline
\end{tabular}


Table 3. Maximum percent variations of spatial displacements with increasing $N_{\mathrm{m}}$.

\begin{tabular}{ccc}
\hline$N_{\mathrm{m}}$ & $x$ displacements & $z$ displacements \\
\hline 10 (reference) & - & - \\
20 & $15.86 \%$ & $17.38 \%$ \\
30 & $6.03 \%$ & $7.15 \%$ \\
40 & $2.06 \%$ & $2.80 \%$ \\
45 & $1.43 \%$ & $1.64 \%$ \\
\hline
\end{tabular}


Table 4. Slug flow parameters at $Q_{\mathrm{G}} / Q_{\mathrm{L}}=3.0$ during the recorded periods in Fig. 9.

\begin{tabular}{|c|c|c|c|c|c|c|}
\hline$t(\mathrm{~s})$ & No. & $L_{\mathrm{s}} / D$ & $U_{\mathrm{t}}(\mathrm{m} / \mathrm{s})$ & $f_{\mathrm{u}}(\mathrm{Hz})$ & $F_{\mathrm{X}}(\mathrm{mN})$ & $F_{\mathrm{Z}}(\mathrm{mN})$ \\
\hline \multirow{2}{*}{75.22} & 1 & 16.00 & 2.441 & 5.90 & 30.77 & 12.98 \\
\hline & 2 & 17.00 & 2.400 & 6.65 & 47.37 & -3.94 \\
\hline \multirow{2}{*}{75.34} & 1 & 17.00 & 2.400 & 6.65 & 32.56 & 13.02 \\
\hline & 2 & 45.00 & 2.526 & 2.65 & 95.46 & -78.78 \\
\hline 75.54 & 1 & 45.00 & 2.526 & 2.65 & 132.71 & -36.77 \\
\hline \multirow{2}{*}{75.74} & 1 & 45.00 & 2.526 & 2.65 & 70.88 & 50.62 \\
\hline & 2 & 45.00 & 2.526 & 2.65 & 95.51 & -30.34 \\
\hline \multirow{2}{*}{75.79} & 1 & 45.00 & 2.526 & 2.65 & 58.80 & 60.03 \\
\hline & 2 & 45.00 & 2.526 & 2.65 & 91.94 & -14.89 \\
\hline \multirow{2}{*}{75.94} & 1 & 45.00 & 2.526 & 2.65 & 112.18 & 6.95 \\
\hline & 2 & 12.00 & 2.441 & 9.03 & 28.38 & -11.72 \\
\hline \multirow{3}{*}{76.18} & 1 & 45.00 & 2.526 & 2.65 & 57.86 & 58.12 \\
\hline & 2 & 12.00 & 2.441 & 9.03 & 36.15 & -1.46 \\
\hline & 3 & 31.00 & 2.400 & 3.70 & 60.41 & -8.23 \\
\hline \multirow{3}{*}{80.98} & 1 & 18.60 & 2.441 & 3.57 & 27.01 & 20.58 \\
\hline & 2 & 19.00 & 2.441 & 4.11 & 44.44 & 0.77 \\
\hline & 3 & 22.20 & 2.441 & 5.22 & 49.84 & -56.64 \\
\hline \multirow{2}{*}{81.13} & 1 & 19.00 & 2.441 & 4.11 & 37.32 & 14.12 \\
\hline & 2 & 22.20 & 2.441 & 5.22 & 62.92 & -25.91 \\
\hline \multirow{3}{*}{81.23} & 1 & 19.00 & 2.441 & 4.11 & 36.73 & 22.57 \\
\hline & 2 & 22.20 & 2.441 & 5.22 & 50.50 & 3.33 \\
\hline & 3 & 22.00 & 2.441 & 3.87 & 52.98 & -44.49 \\
\hline \multirow{3}{*}{81.41} & 1 & 22.20 & 2.441 & 5.22 & 49.68 & 4.36 \\
\hline & 2 & 22.00 & 2.441 & 3.87 & 51.52 & 0.22 \\
\hline & 3 & 17.00 & 2.400 & 6.65 & 30.37 & -40.93 \\
\hline \multirow{3}{*}{81.64} & 1 & 22.00 & 2.441 & 3.87 & 39.16 & 22.30 \\
\hline & 2 & 18.60 & 2.441 & 5.65 & 45.72 & -3.04 \\
\hline & 3 & 14.40 & 2.441 & 8.80 & 35.03 & -48.51 \\
\hline \multirow{4}{*}{81.86} & 1 & 18.60 & 2.441 & 5.65 & 36.89 & 15.06 \\
\hline & 2 & 14.40 & 2.441 & 8.80 & 33.73 & -0.71 \\
\hline & 3 & 16.50 & 2.400 & 6.67 & 47.60 & -27.95 \\
\hline & 4 & 16.00 & 2.441 & 5.90 & 32.27 & -37.30 \\
\hline \multirow{4}{*}{82.05} & 1 & 14.40 & 2.441 & 8.80 & 25.49 & 13.21 \\
\hline & 2 & 16.50 & 2.400 & 6.67 & 33.20 & 10.21 \\
\hline & 3 & 16.00 & 2.441 & 5.90 & 42.16 & -14.07 \\
\hline & 4 & 21.00 & 2.440 & 5.13 & 44.15 & -57.12 \\
\hline
\end{tabular}


Table 5. Slug flow parameters in the riser at different gas-to-liquid flow rate ratios.

\begin{tabular}{ccccccc}
\hline$Q_{\mathrm{G}} / Q_{\mathrm{L}}$ & $L_{\mathrm{u}} / D$ & $L_{\mathrm{s}} / D$ & $L_{\mathrm{g}} / D$ & $H_{\mathrm{s}}$ & $U_{\mathrm{t}}(\mathrm{m} / \mathrm{s})$ & $f_{\mathrm{u}}(\mathrm{Hz})$ \\
\hline 1.0 & $12.33-36.00$ & $6.00-24.33$ & $6.00-13.67$ & $0.486-0.774$ & $1.152-1.171$ & $5.38-13.01$ \\
1.5 & $16.67-51.00$ & $6.67-30.00$ & $7.00-26.80$ & $0.208-0.800$ & $1.485-1.500$ & $4.85-13.89$ \\
2.0 & $25.00-93.00$ & $6.67-24.34$ & $11.67-70.20$ & $0.215-0.588$ & $1.846-1.870$ & $3.35-10.39$ \\
2.5 & $44.00-156.00$ & $6.67-41.67$ & $24.00-118.80$ & $0.200-0.526$ & $2.087-2.149$ & $2.30-9.80$ \\
3.0 & $45.00-216.00$ & $13.80-48.00$ & $12.00-168.00$ & $0.161-0.500$ & $2.400-2.571$ & $1.98-9.03$ \\
3.5 & $54.00-276.00$ & $11.67-55.20$ & $24.00-220.80$ & $0.138-0.654$ & $2.717-3.00$ & $1.64-8.25$ \\
4.0 & $67.20-318.80$ & $12.33-57.00$ & $39.00-282.00$ & $0.110-0.543$ & $3.000-3.200$ & $1.60-7.06$ \\
4.5 & $84.00-351.00$ & $15.60-75.00$ & $48.00-311.00$ & $0.106-0.529$ & $3.273-3.692$ & $1.59-6.80$ \\
\hline
\end{tabular}


Table 6. Slug flow parameters for the longest slug unit at different gas-to-liquid flow rate ratios.

\begin{tabular}{ccccccc}
\hline$Q_{\mathrm{G}} / Q_{\mathrm{L}}$ & $L_{\mathrm{u}, \max } / D$ & $L_{\mathrm{s}} / D$ & $L_{\mathrm{g}} / D$ & $H_{\mathrm{s}}$ & $U_{\mathrm{t}}(\mathrm{m} / \mathrm{s})$ & $f_{\mathrm{u}, \min }(\mathrm{Hz})$ \\
\hline 1.0 & 36.00 & 24.00 & 12.00 & 0.667 & 1.161 & 5.38 \\
1.5 & 51.00 & 24.20 & 26.80 & 0.475 & 1.485 & 4.85 \\
2.0 & 93.00 & 22.80 & 70.20 & 0.245 & 1.870 & 3.35 \\
2.5 & 156.00 & 37.20 & 118.80 & 0.239 & 2.149 & 2.30 \\
3.0 & 216.00 & 48.00 & 168.00 & 0.222 & 2.571 & 1.98 \\
3.5 & 276.00 & 55.20 & 220.80 & 0.200 & 2.717 & 1.64 \\
4.0 & 318.80 & 36.00 & 282.00 & 0.113 & 3.064 & 1.60 \\
4.5 & 351.00 & 40.00 & 311.00 & 0.114 & 3.348 & 1.59 \\
\hline
\end{tabular}


Table 7. Interrelationships between slug flow hydrodynamics and riser vibrations.

\begin{tabular}{|c|c|c|c|c|c|c|c|c|}
\hline$Q_{\mathrm{G}} / Q_{\mathrm{L}}$ & $h_{\mathrm{s}}$ & $A_{\mathrm{X}, \max } / D$ & $A_{\mathrm{Z}, \text { max }} / D$ & No. & $L_{\mathrm{s}} / D$ & Location $(s / l)$ & $U_{\mathrm{t}}$ & $F(\mathrm{mN})$ \\
\hline \multirow{19}{*}{1.0} & \multirow{19}{*}{0.557} & \multirow{19}{*}{0.60} & \multirow{19}{*}{0.47} & 1 & 6.00 & $0.975-1.000$ & 1.152 & 7.81 \\
\hline & & & & 2 & 6.30 & $0.936-0.963$ & 1.171 & 4.56 \\
\hline & & & & 3 & 6.30 & $0.886-0.913$ & 1.171 & 4.99 \\
\hline & & & & 4 & 6.00 & $0.838-0.863$ & 1.171 & 18.76 \\
\hline & & & & 5 & 6.00 & $0.788-0.813$ & 1.161 & 8.78 \\
\hline & & & & 6 & 6.00 & $0.738-0.763$ & 1.171 & 4.99 \\
\hline & & & & 7 & 5.40 & $0.665-0.688$ & 1.161 & 9.99 \\
\hline & & & & 8 & 6.10 & $0.637-0.663$ & 1.161 & 12.42 \\
\hline & & & & 9 & 6.20 & $0.612-0.638$ & 1.171 & 9.09 \\
\hline & & & & 10 & 6.40 & $0.560-0.588$ & 1.171 & 21.98 \\
\hline & & & & 11 & 6.10 & $0.512-0.538$ & 1.171 & 3.27 \\
\hline & & & & 12 & 6.00 & $0.463-0.488$ & 1.160 & 11.21 \\
\hline & & & & 13 & 6.40 & $0.410-0.438$ & 1.161 & 19.57 \\
\hline & & & & 14 & 6.00 & $0.360-0.385$ & 1.161 & 11.77 \\
\hline & & & & 15 & 6.00 & $0.284-0.309$ & 1.156 & 20.18 \\
\hline & & & & 16 & 24.33 & $0.161-0.261$ & 1.142 & 62.60 \\
\hline & & & & 17 & 6.00 & $0.111-0.136$ & 1.151 & 15.53 \\
\hline & & & & 18 & 6.40 & $0.060-0.088$ & 1.161 & 21.42 \\
\hline & & & & 19 & 6.00 & $0.038-0.063$ & 1.158 & 11.40 \\
\hline \multirow{12}{*}{1.5} & \multirow{12}{*}{0.457} & \multirow{12}{*}{1.11} & \multirow{12}{*}{0.80} & 1 & 12.00 & $0.888-0.938$ & 1.484 & 9.45 \\
\hline & & & & 2 & 3.00 & $0.850-0.863$ & 1.500 & 6.89 \\
\hline & & & & 3 & 4.80 & $0.817-0.838$ & 1.484 & 8.76 \\
\hline & & & & 4 & 6.00 & $0.565-0.588$ & 1.494 & 21.88 \\
\hline & & & & 5 & 9.00 & $0.650-0.688$ & 1.500 & 8.67 \\
\hline & & & & 6 & 4.20 & $0.620-0.638$ & 1.484 & 9.69 \\
\hline & & & & 7 & 5.40 & $0.565-0.588$ & 1.484 & 6.50 \\
\hline & & & & 8 & 30.00 & $0.350-0.475$ & 1.484 & 51.61 \\
\hline & & & & 9 & 6.00 & $0.267-0.288$ & 1.500 & 34.59 \\
\hline & & & & 10 & 14.00 & $0.193-0.238$ & 1.484 & 42.58 \\
\hline & & & & 11 & 11.00 & $0.092-0.138$ & 1.500 & 32.96 \\
\hline & & & & 12 & 4.20 & $0.020-0.038$ & 1.484 & 4.28 \\
\hline \multirow{6}{*}{2.0} & \multirow{6}{*}{0.349} & \multirow{6}{*}{1.83} & \multirow{6}{*}{1.85} & 1 & 24.34 & $0.835-0.938$ & 1.870 & 28.75 \\
\hline & & & & 2 & 9.00 & $0.650-0.688$ & 1.846 & 20.07 \\
\hline & & & & 3 & 6.00 & $0.836-0.863$ & 1.846 & 21.77 \\
\hline & & & & 4 & 12.00 & $0.438-0.488$ & 1.894 & 30.67 \\
\hline & & & & 5 & 7.20 & $0.583-0.613$ & 1.870 & 21.59 \\
\hline & & & & 6 & 24.00 & $0.383-0.488$ & 1.870 & 47.74 \\
\hline \multirow{5}{*}{2.5} & \multirow{5}{*}{0.349} & \multirow{5}{*}{3.17} & \multirow{5}{*}{2.12} & 1 & 41.67 & $0.683-0.857$ & 2.118 & 63.90 \\
\hline & & & & 2 & 6.00 & $0.625-0.650$ & 2.118 & 36.30 \\
\hline & & & & 3 & 12.00 & $0.411-0.461$ & 2.148 & 22.81 \\
\hline & & & & 4 & 18.10 & $0.112-0.188$ & 2.128 & 80.76 \\
\hline & & & & 5 & 6.05 & $0.037-0.062$ & 2.149 & 13.34 \\
\hline 3.0 & 0.200 & 4.59 & 3.82 & 1 & 48.00 & $0.744-0.944$ & 2.526 & 99.18 \\
\hline 3.5 & 0.230 & 5.20 & 3.48 & 1 & 55.20 & $0.683-0.913$ & 2.823 & 119.70 \\
\hline \multirow{2}{*}{4.0} & \multirow{2}{*}{0.288} & \multirow{2}{*}{6.47} & \multirow{2}{*}{3.09} & 1 & 57.00 & $0.750-0.988$ & 3.000 & 159.75 \\
\hline & & & & 2 & 12.30 & $0.426-0.478$ & 2.987 & 31.20 \\
\hline 4.5 & 0.338 & 7.32 & 3.81 & 1 & 75.00 & $0.625-0.938$ & 3.273 & 236.41 \\
\hline & & & & 2 & 6.00 & $0.575-0.600$ & 3.270 & 29.39 \\
\hline
\end{tabular}




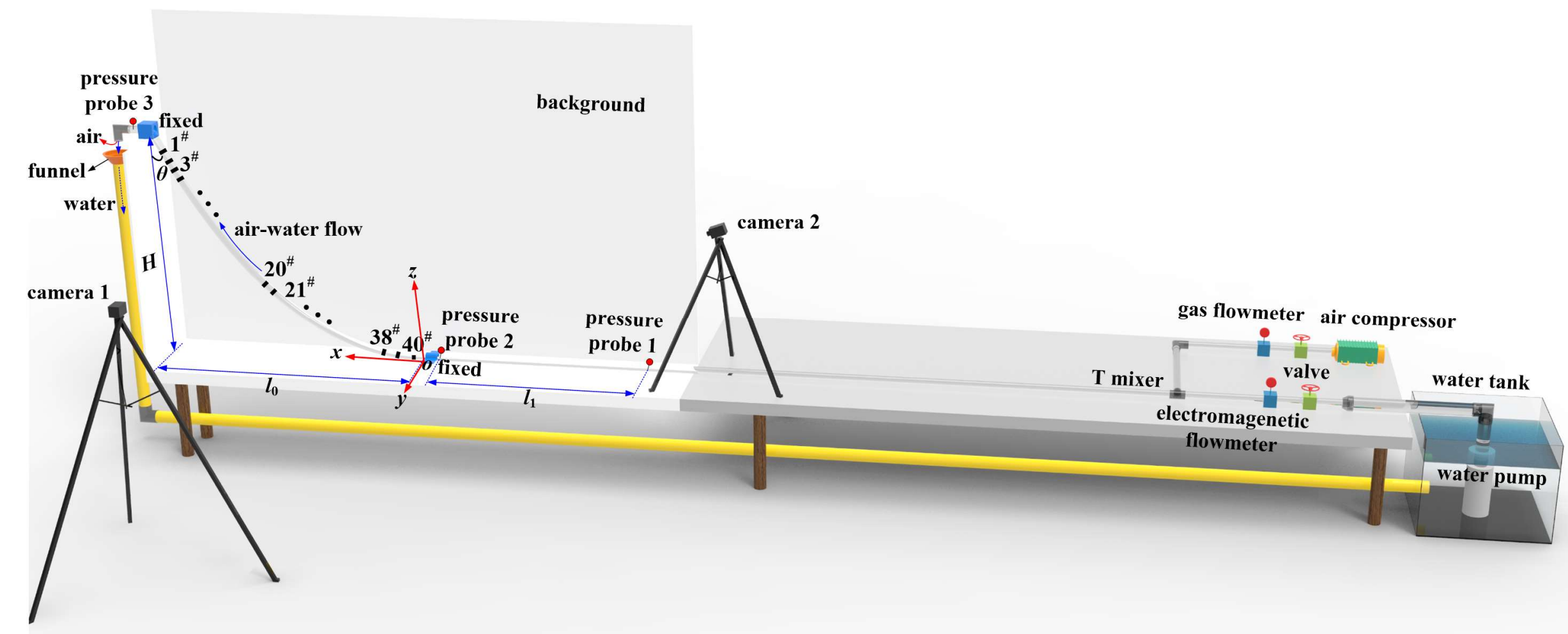

Fig. 1. Experimental setup for slug flow-induced vibration of catenary riser. 


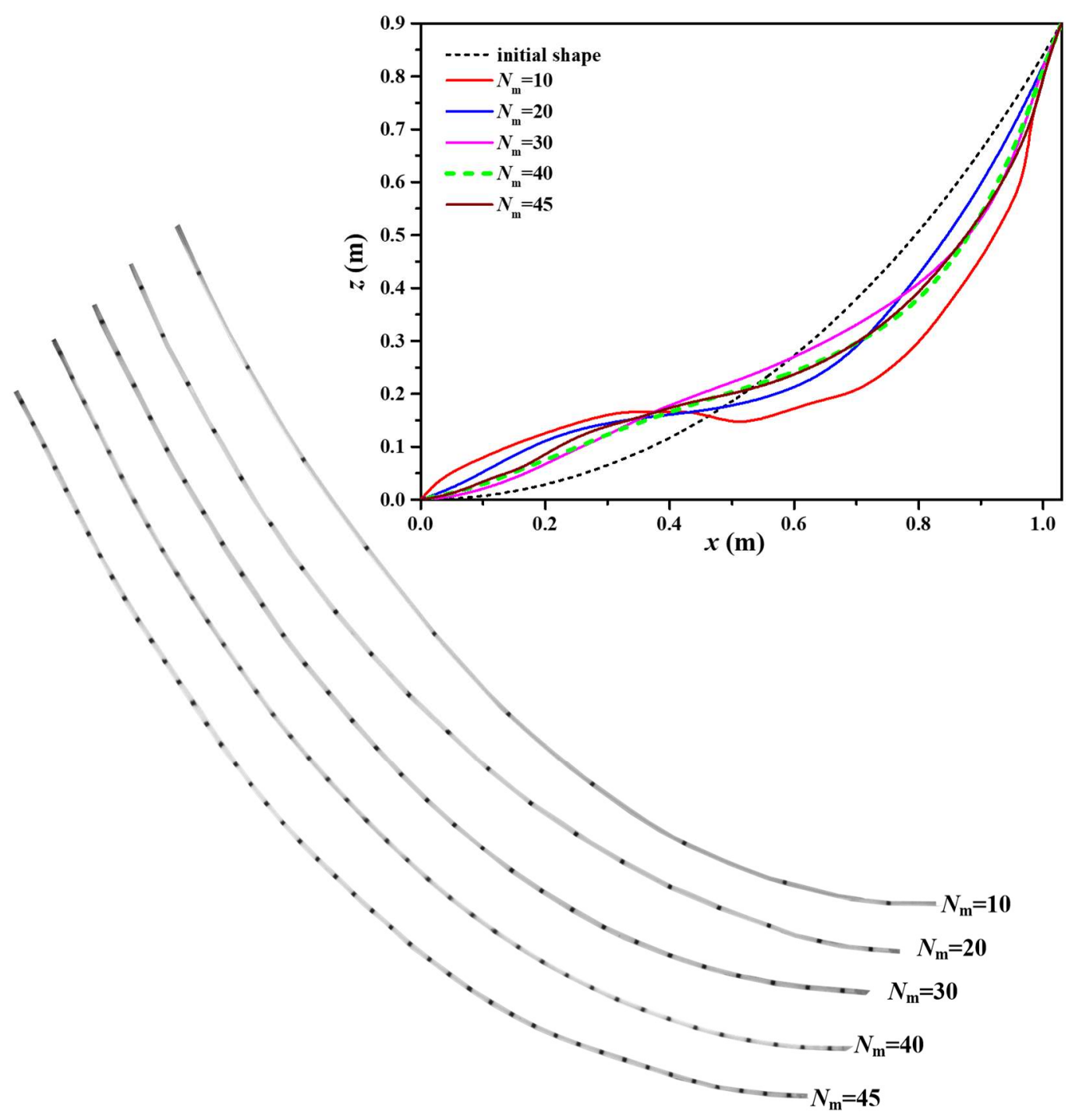

Fig. 2. Variations of riser displacement profiles with the increased number of markers $N_{\mathrm{m}}$ used in experimental measurement. 


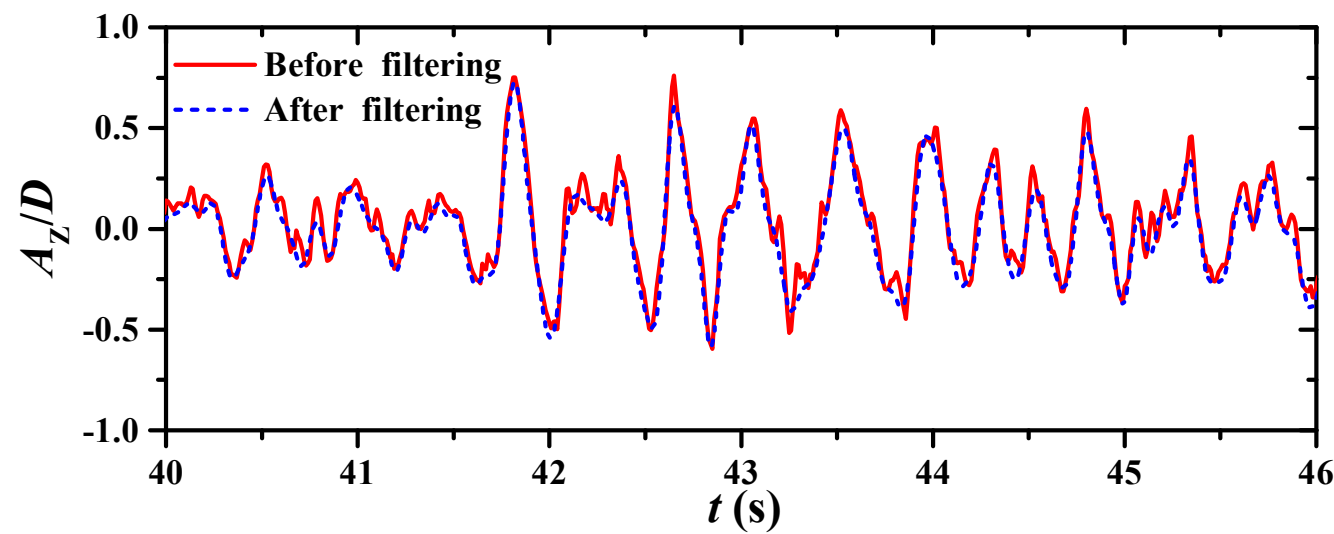

Fig. 3. Time histories of dimensionless vertical response amplitudes before and after the band-pass filtering. 

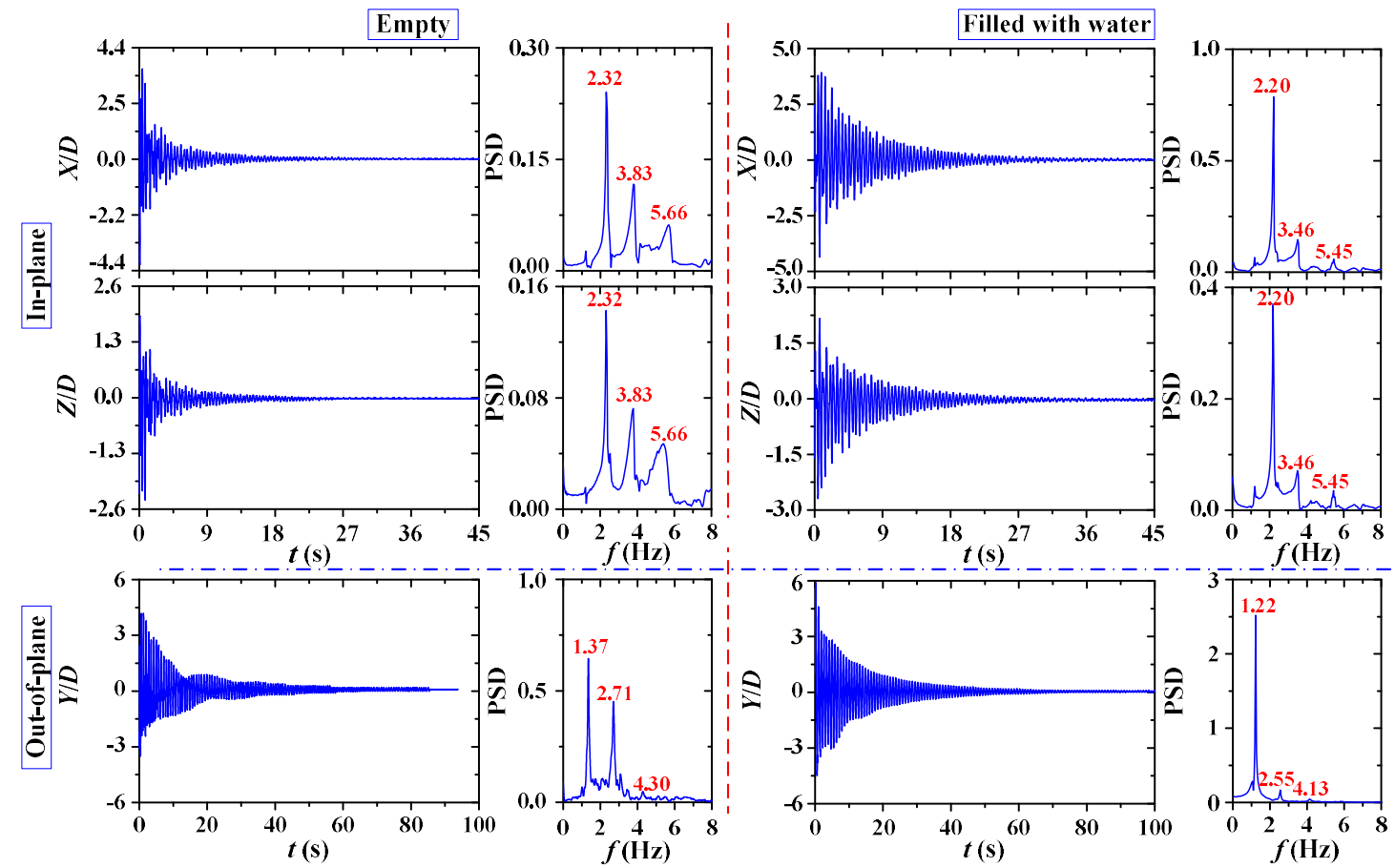

Fig. 4. Free decay test results: $x$ and $z$ denote in-plane response components; $y$ denotes out-of-plane response. 


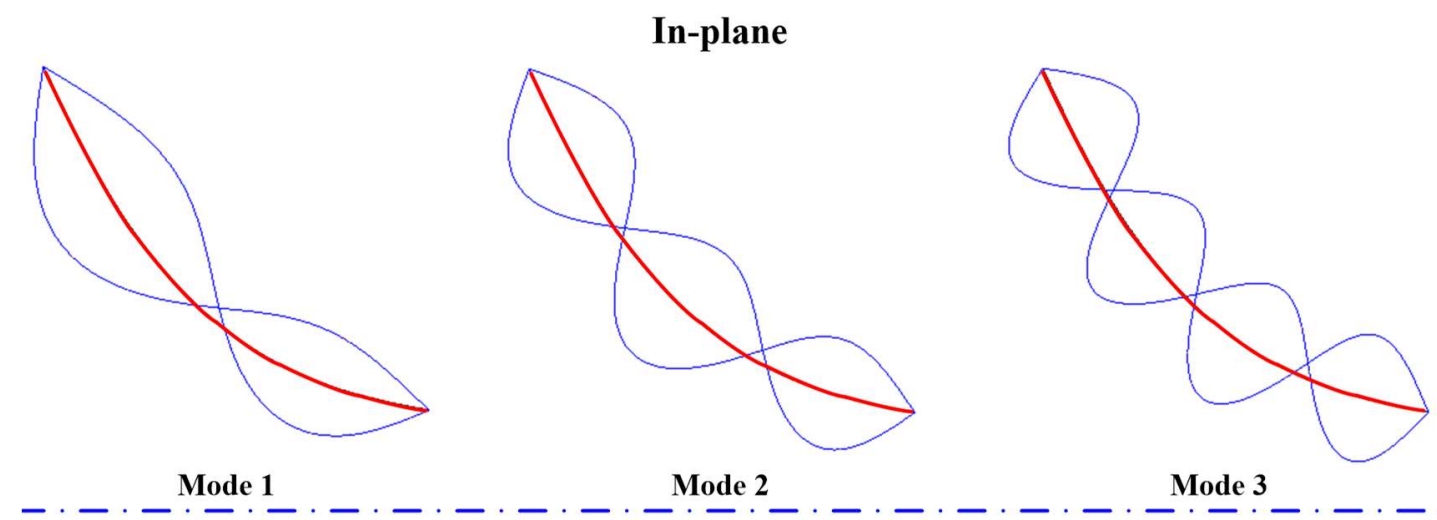

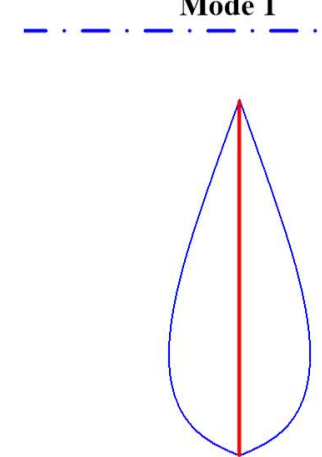

Mode 1

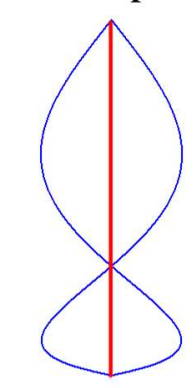

Mode 2

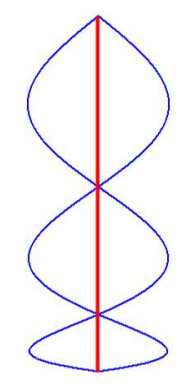

Mode 3

Fig. 5. First three in-plane and out-of-plane modal shapes of catenary riser. 


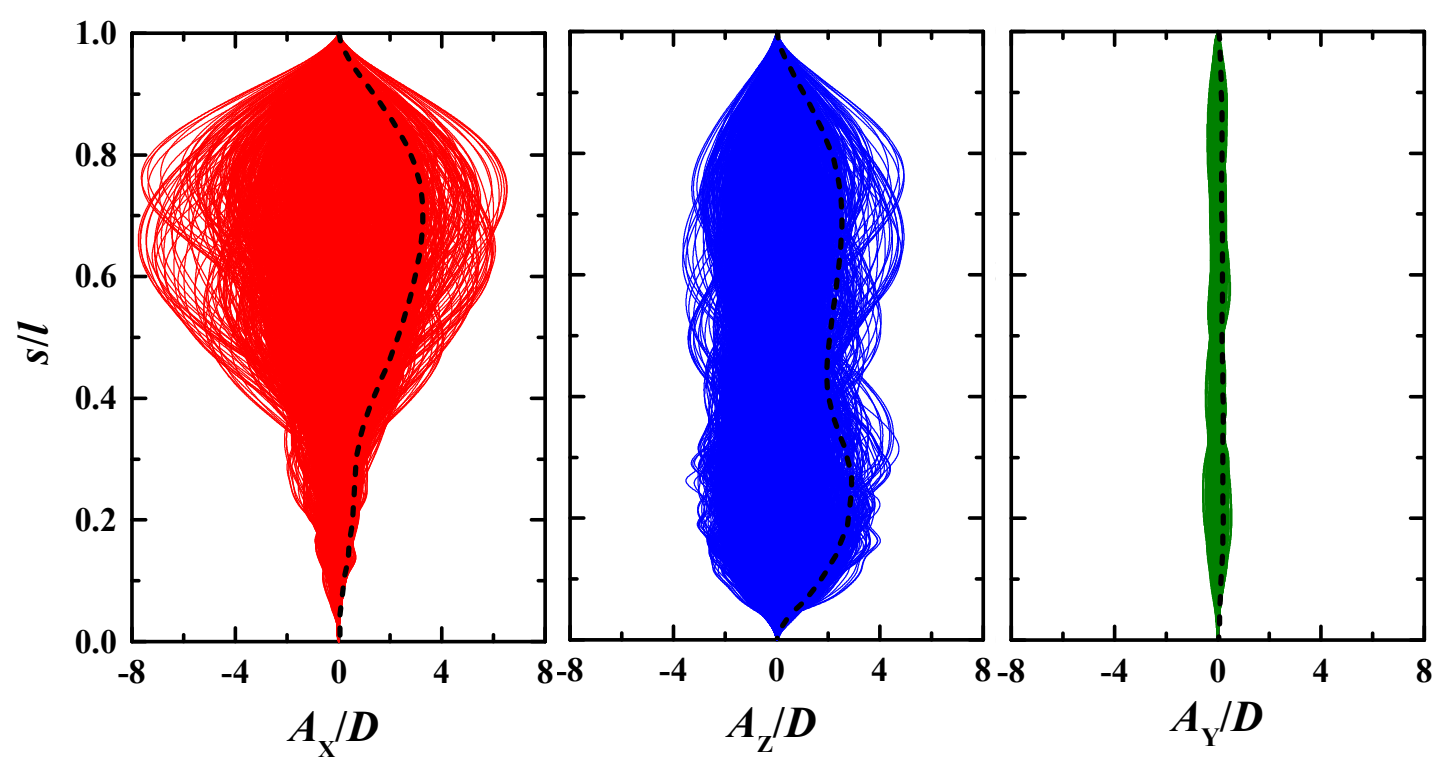

(a)

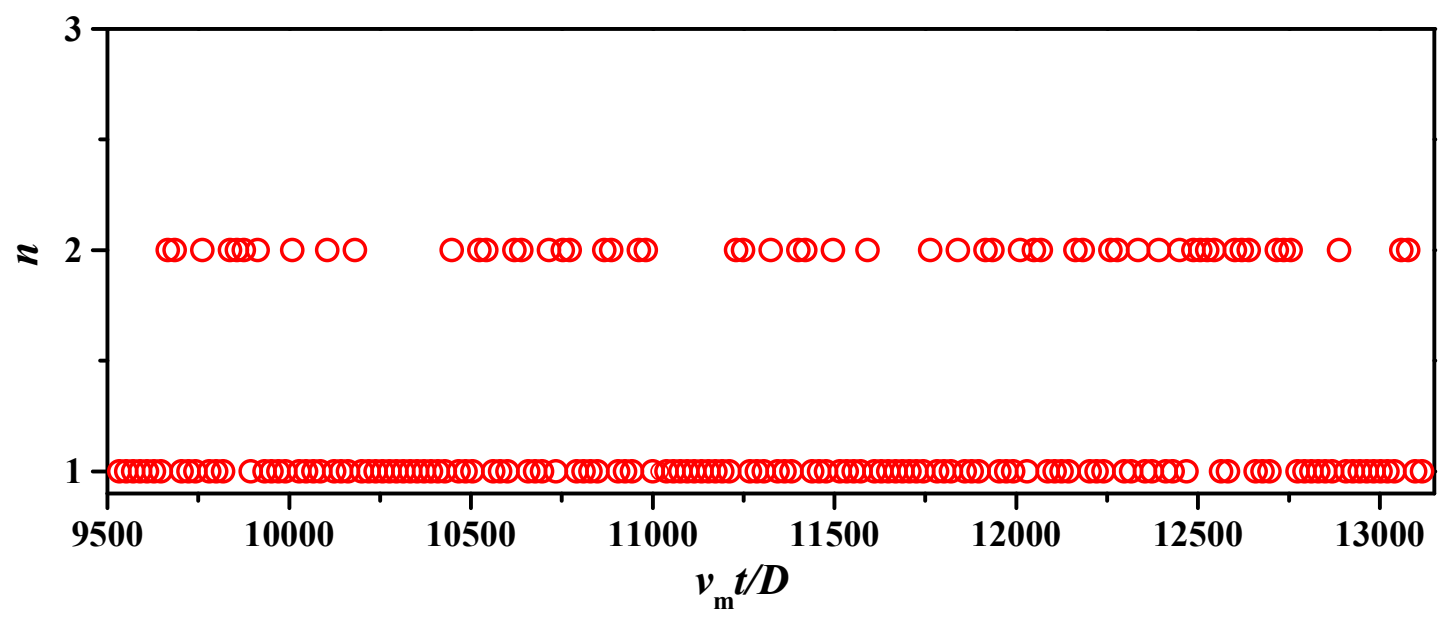

(b)

Fig. 6. Space-time varying response amplitudes at $Q_{\mathrm{G}} / Q_{\mathrm{L}}=3.0$ : (a) spanwise evolution (dashed line represents RMS amplitudes), where $s / l$ denotes the dimensionless position along the riser length; (b) mode switching in time ( $n$ is the mode number). 


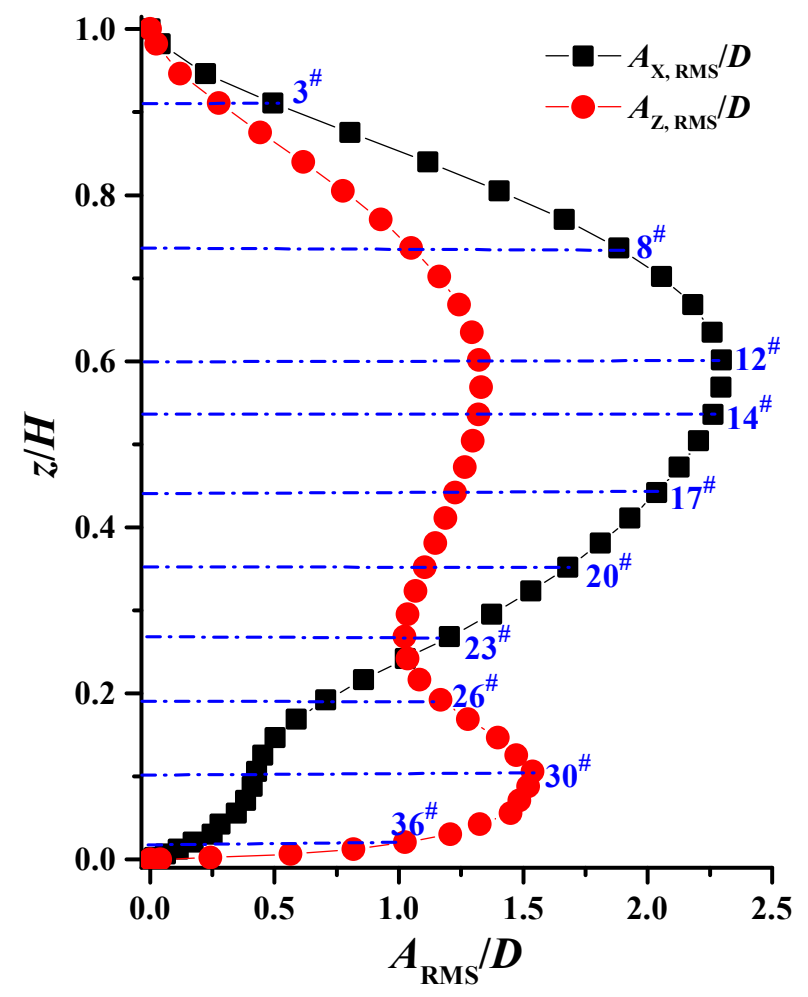

(a)
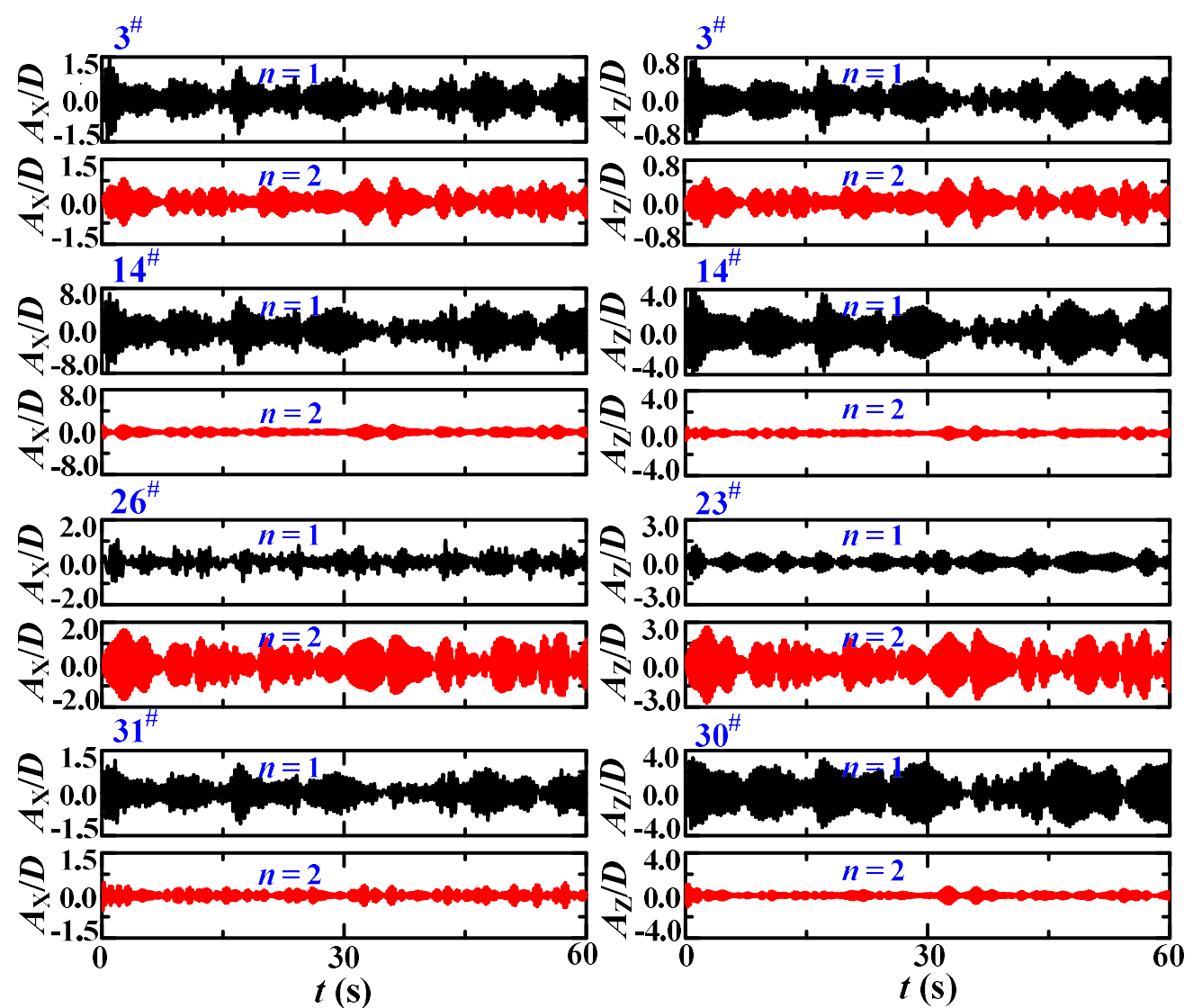

(b)

Fig. 7. Riser responses at $Q_{\mathrm{G}} / Q_{\mathrm{L}}=3.0$ : (a) root-mean-squared amplitudes; (b) decomposed modal amplitudes at representative markers ( $n$ is the mode number). 

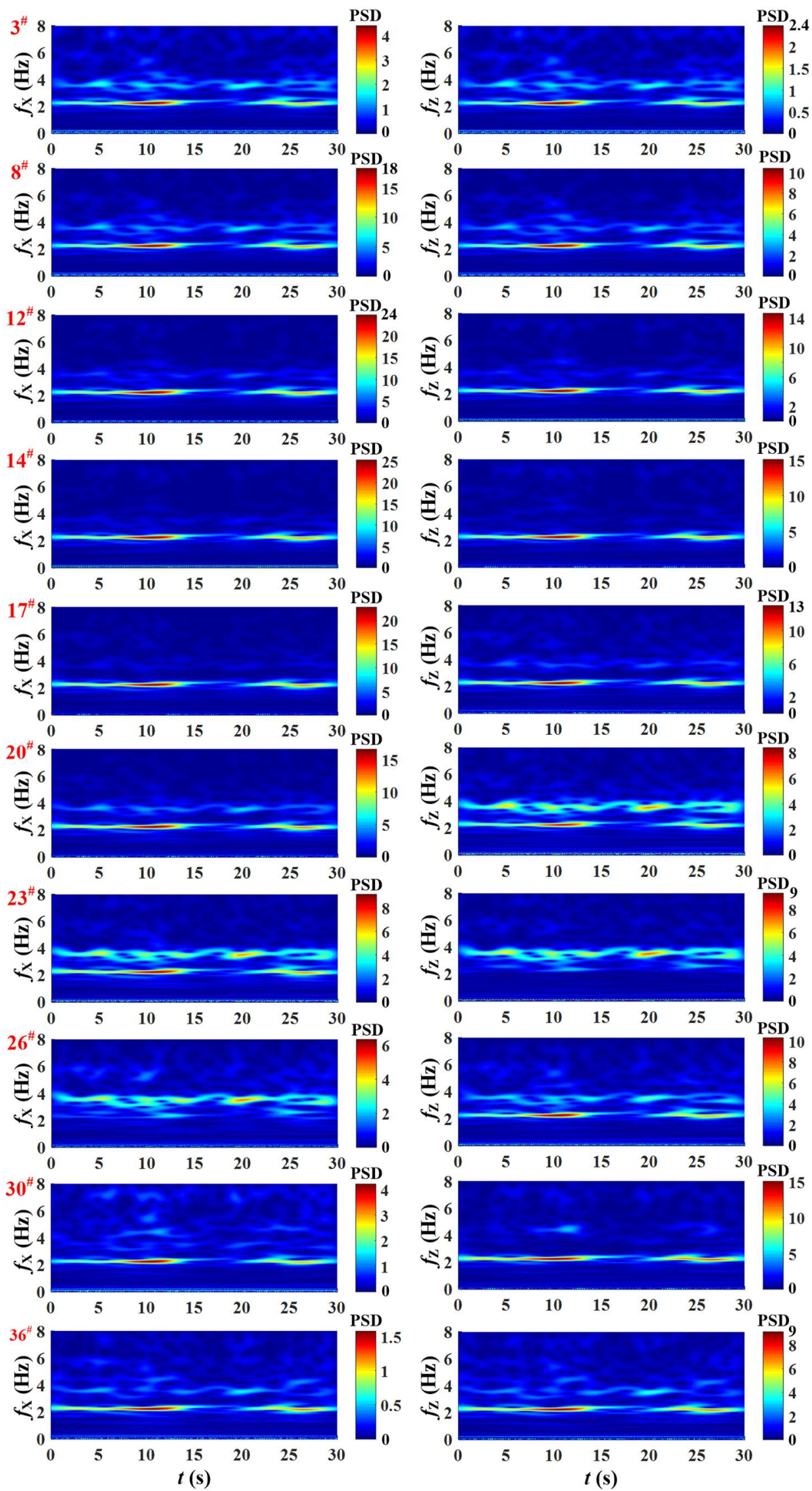

Fig. 8. Time-varying frequencies of in-plane vibration responses at representative markers for $Q_{\mathrm{G}} / Q_{\mathrm{L}}=3.0$ 

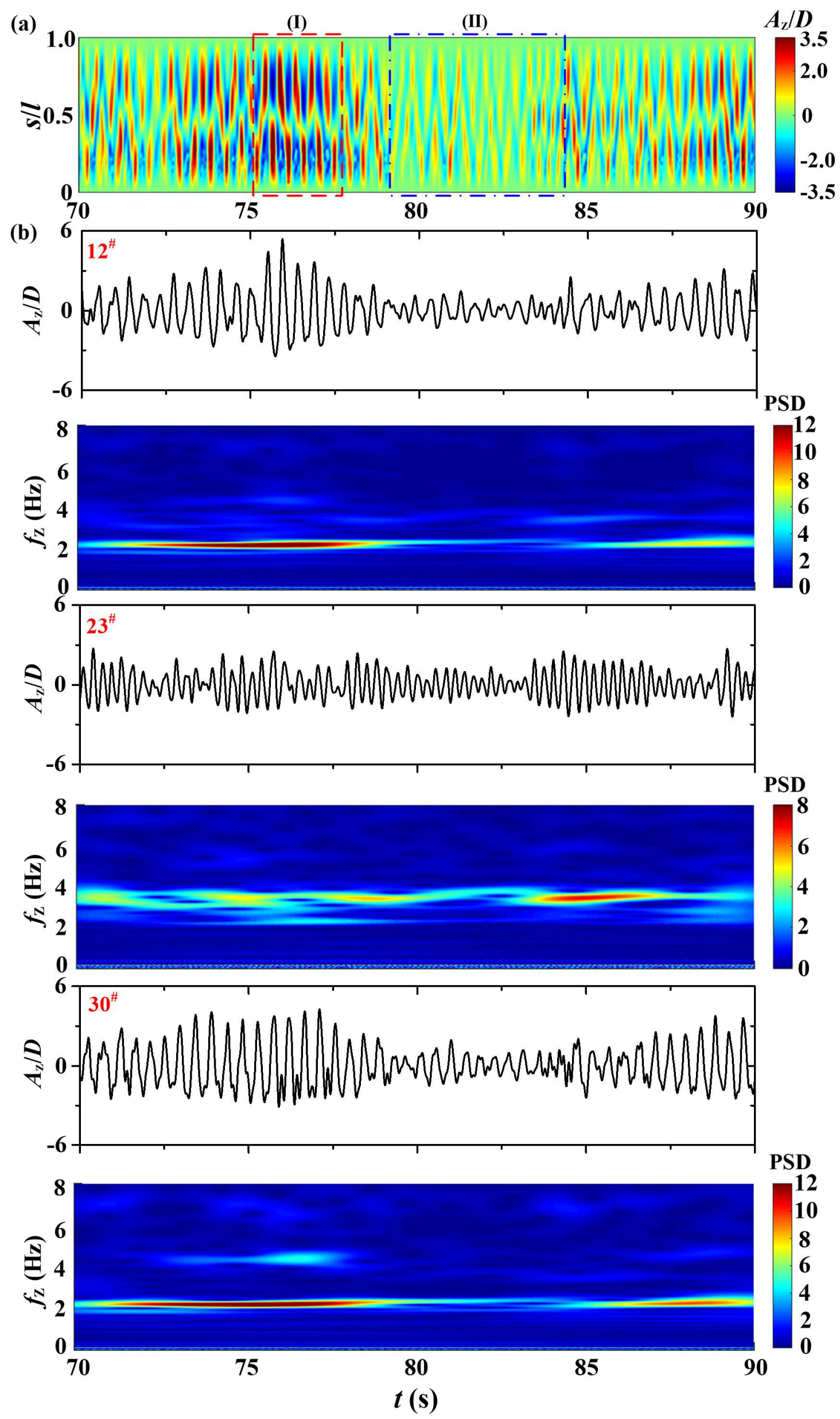


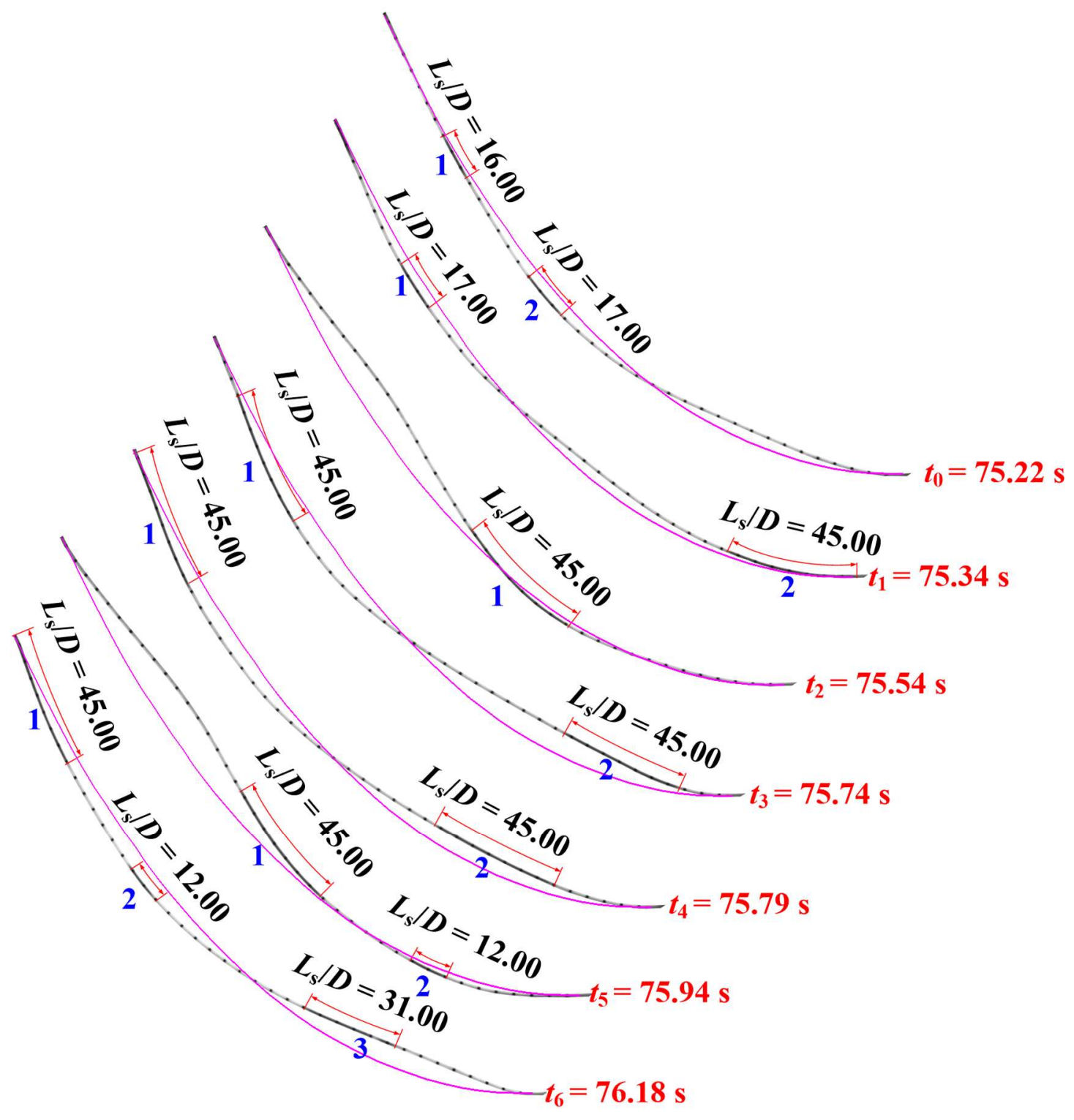

(c) 


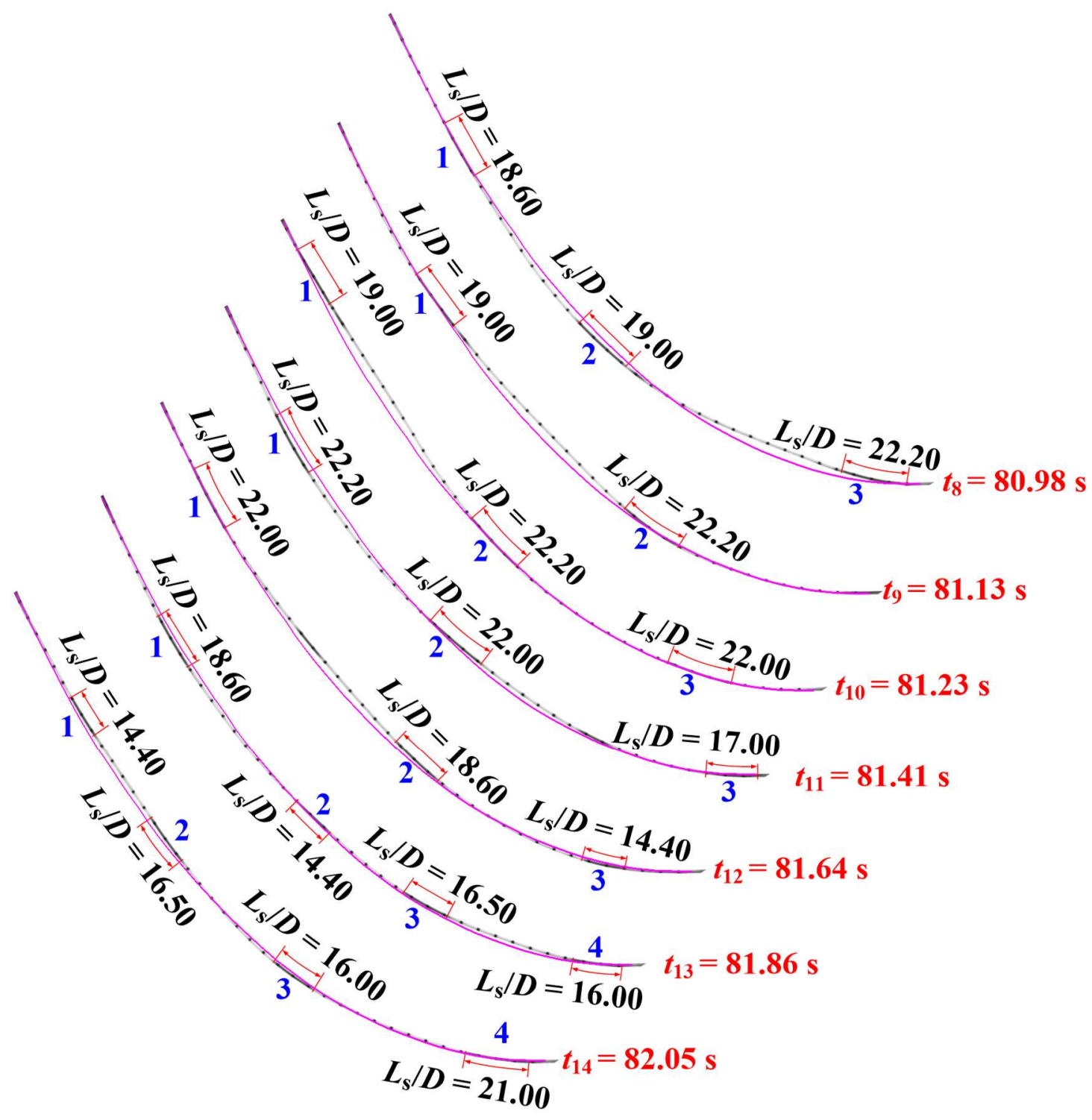

(d)

Fig. 9. Mode switching over time and the corresponding slug flow information at $Q_{\mathrm{G}} / Q_{\mathrm{L}}=3.0$ : (a) evolution of amplitudes along $z$ direction; (b) variations of amplitudes and frequencies over time at markers $12^{\#}, 23^{\#}$ and $30^{\#}$; instantaneous slug flow information during first (c) and second (d) periods identified by I and II in (a). 


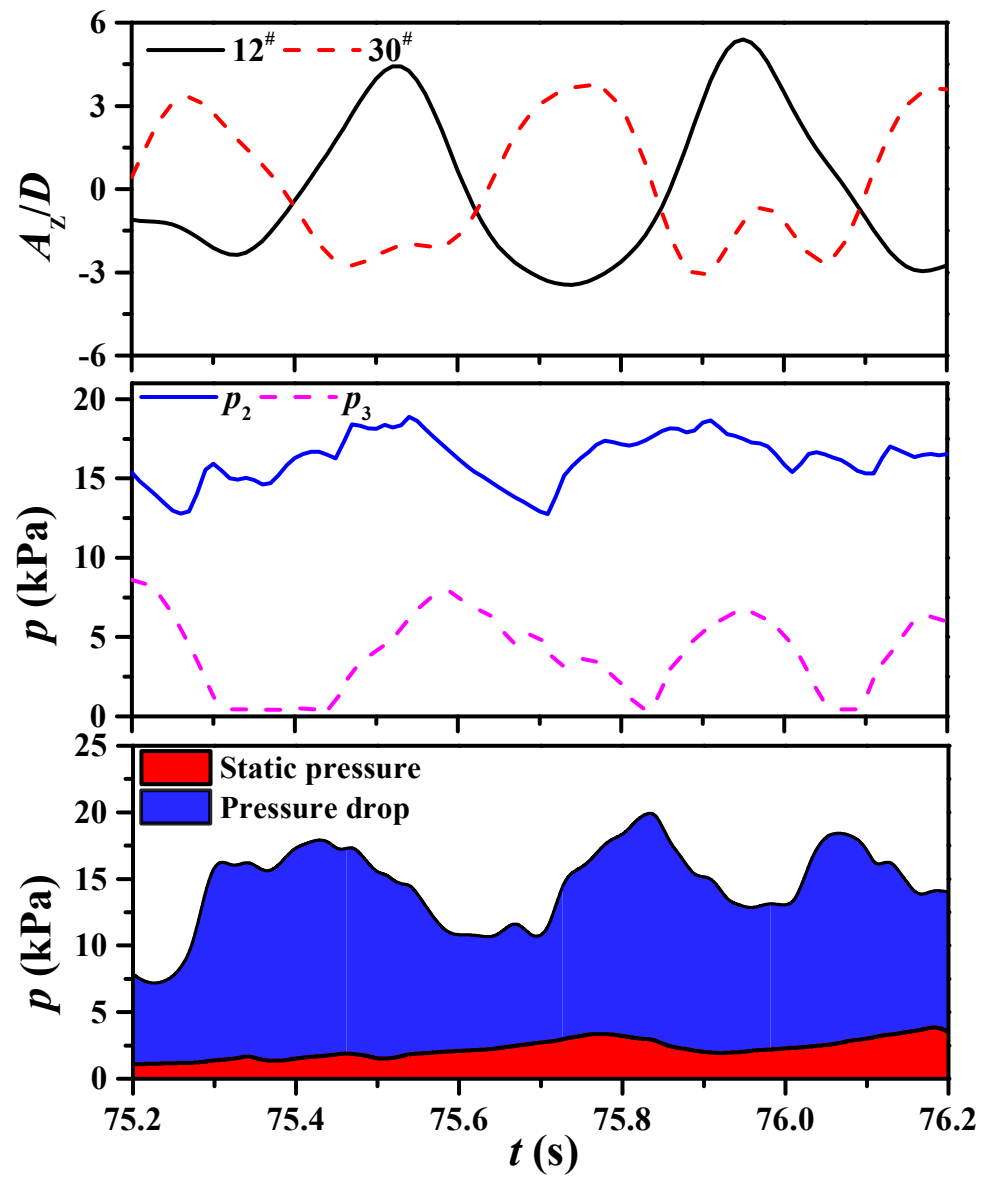

Fig. 10. Variations of vertical responses, monitored pressure at the riser base and top, associated pressure drop and static pressure due to the liquid column in the riser, during the first period I marked in Fig.9a. 


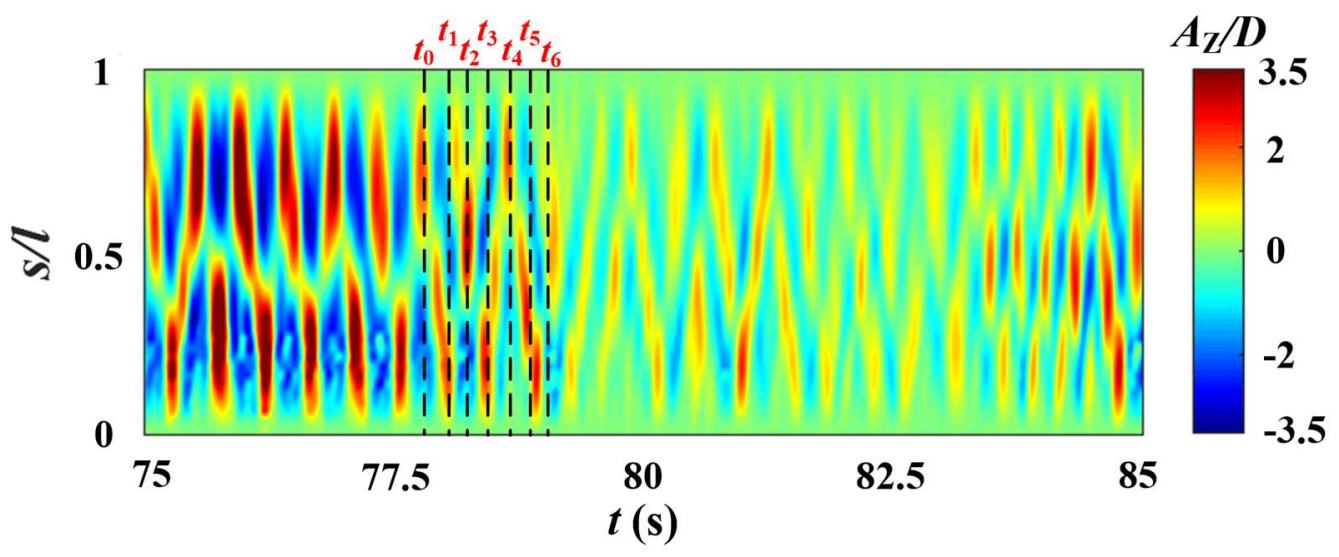

(a)

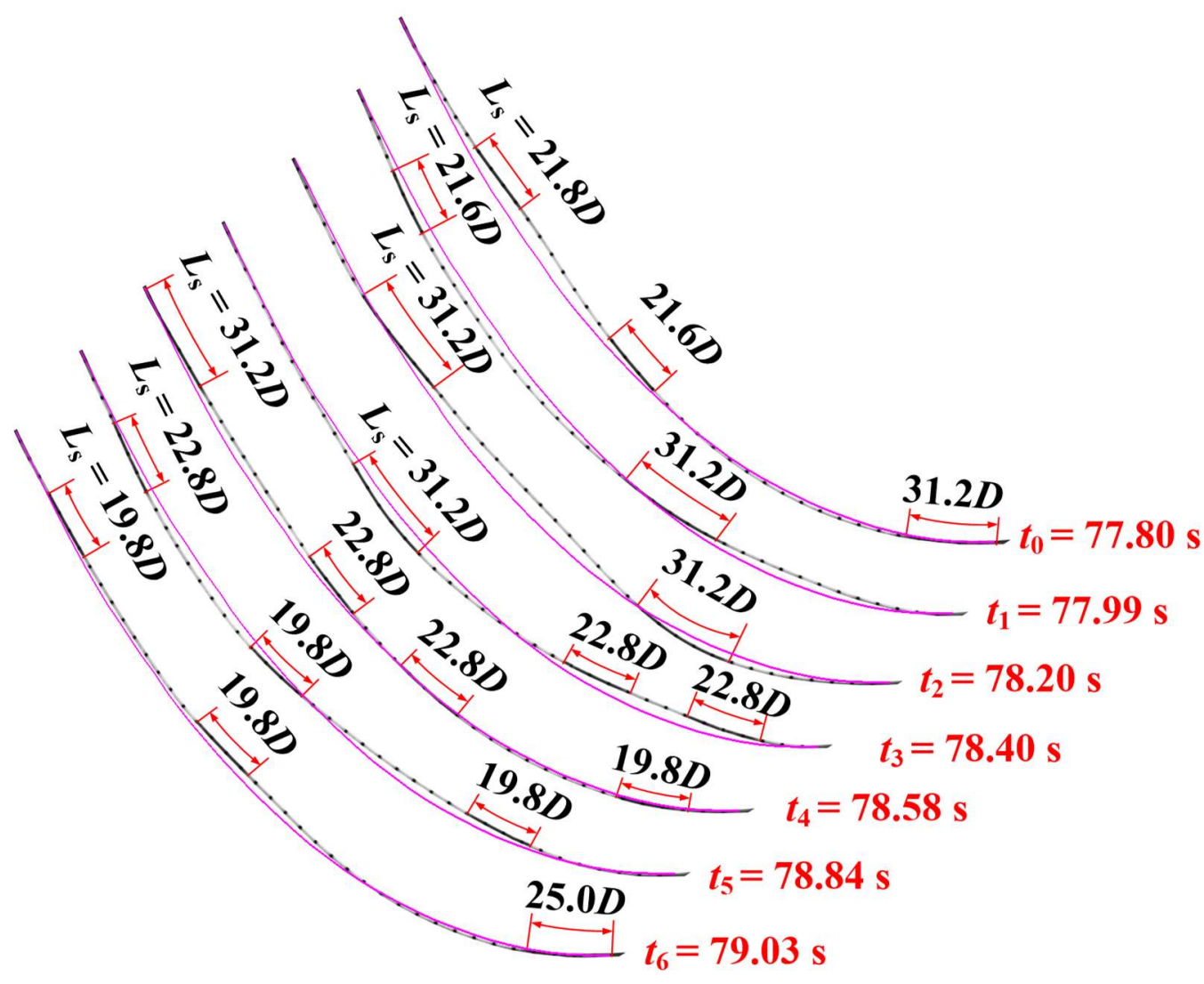

(b)

Fig. 11. Transition from standing wave to travelling wave at $Q_{\mathrm{G}} / Q_{\mathrm{L}}=3.0$ : (a) space-time varying amplitude contours over $10 \mathrm{~s}$; (b) the associated slug flow information in the riser. 


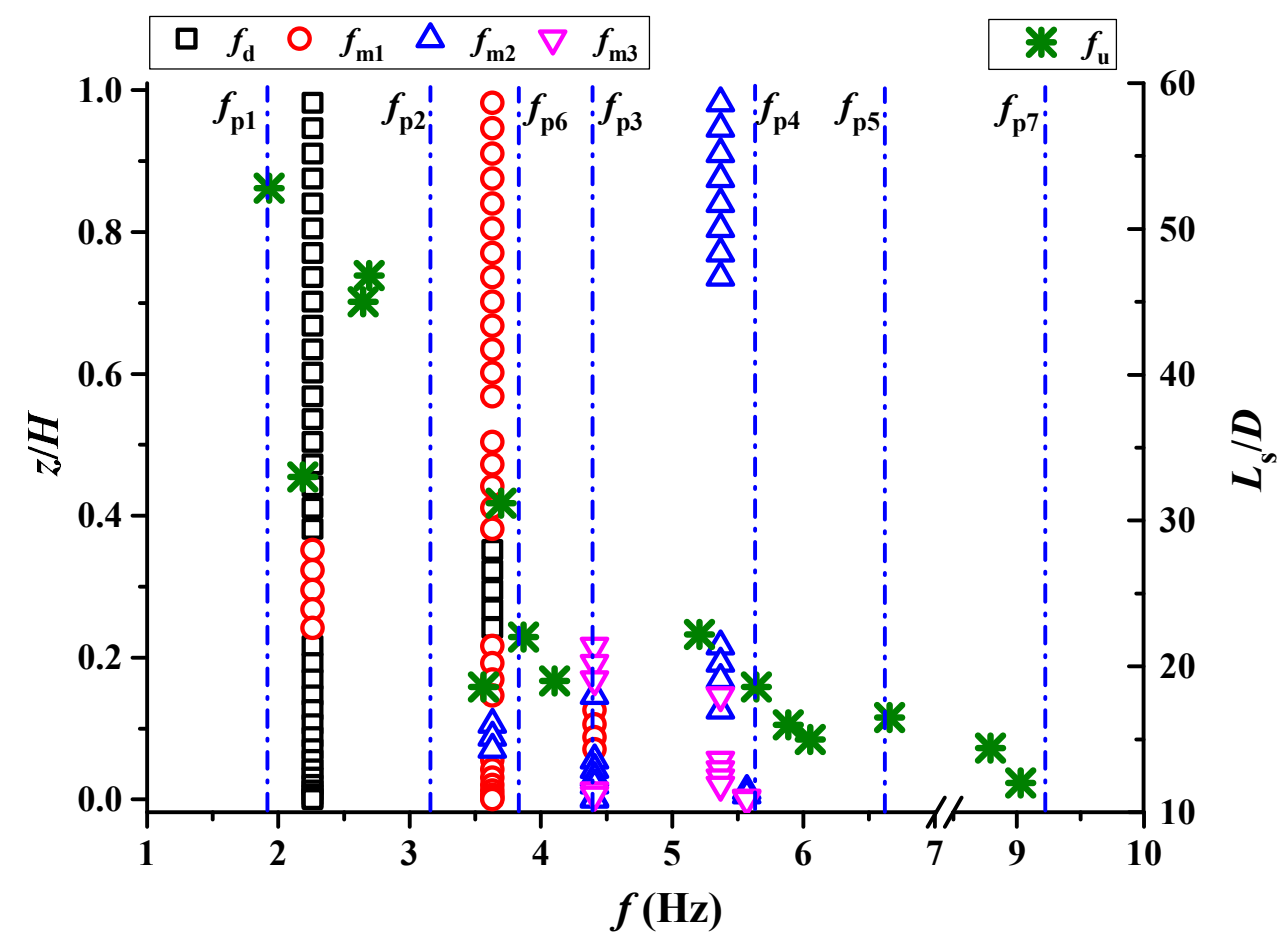

(a)
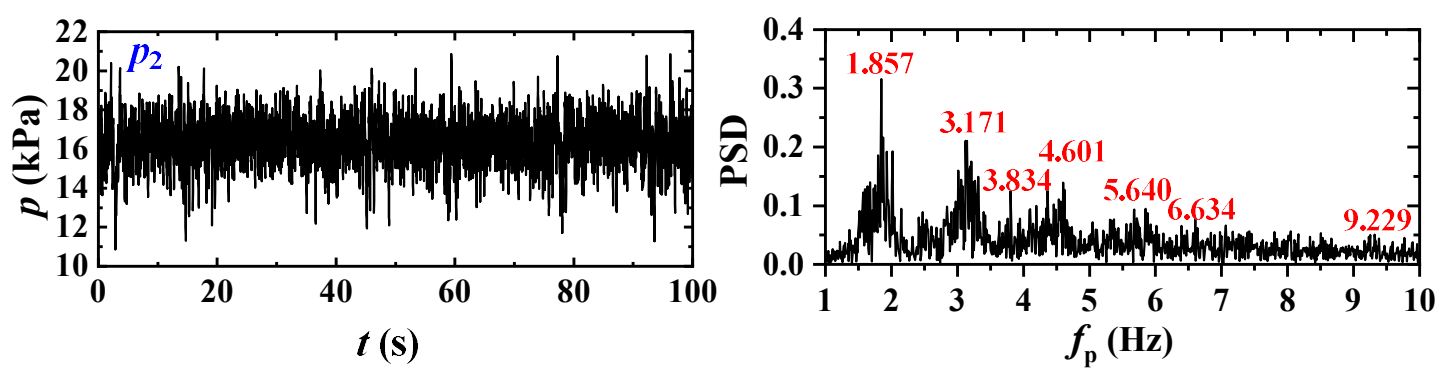

(b)

Fig. 12. (a) Comparison of vibration frequencies, pressure fluctuation frequencies and slug flow frequencies at $Q_{\mathrm{G}} / Q_{\mathrm{L}}=3.0$. (b) Pressure fluctuation at riser base and corresponding frequencies. 

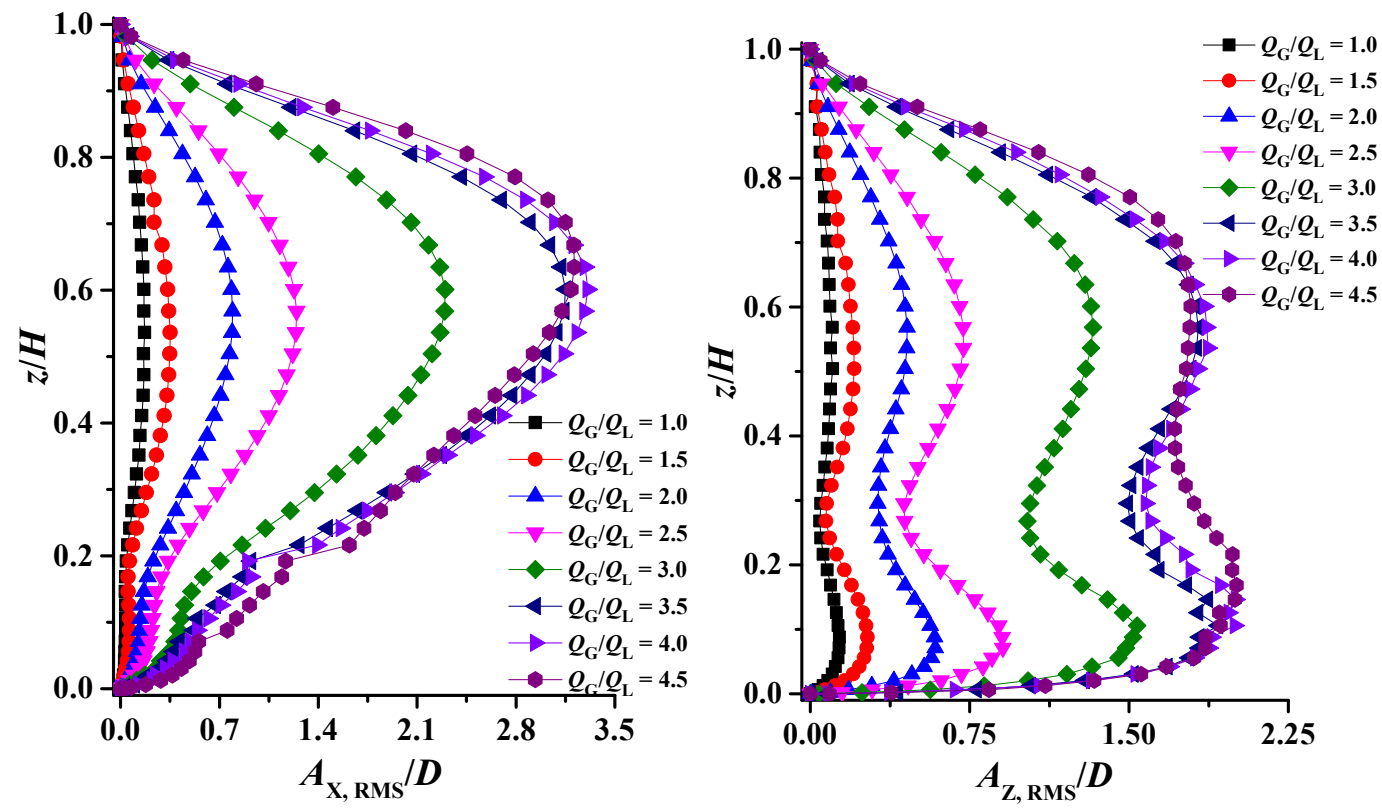

Fig. 13. Spanwise evolution of RMS amplitudes at different gas-to-liquid flow rate ratios. 

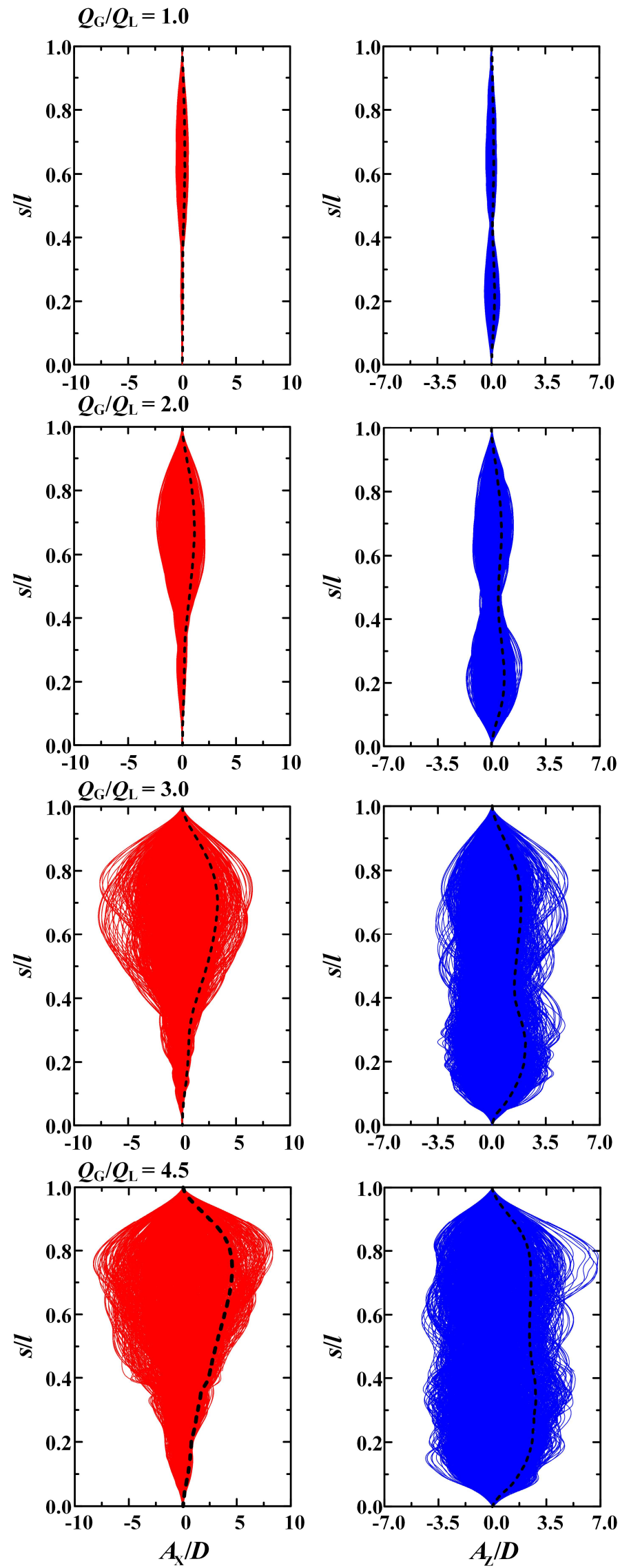

Fig. 14. Instantaneous profiles of catenary riser SIV at representative gas-to-liquid flow rate ratios. 

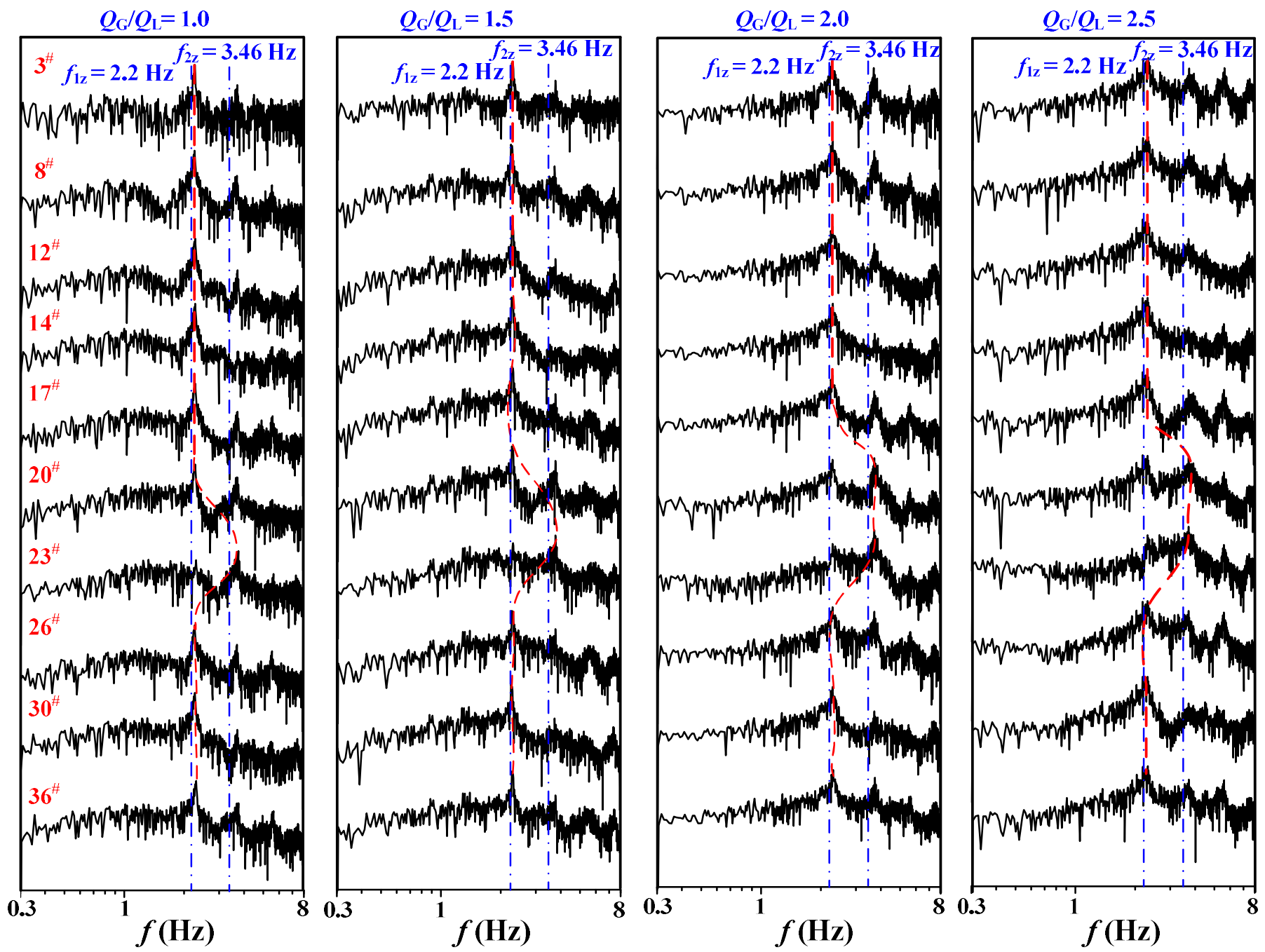

Fig. 15. Vibration frequency spectra in the $z$ direction at different gas-to-liquid flow rate ratios. 

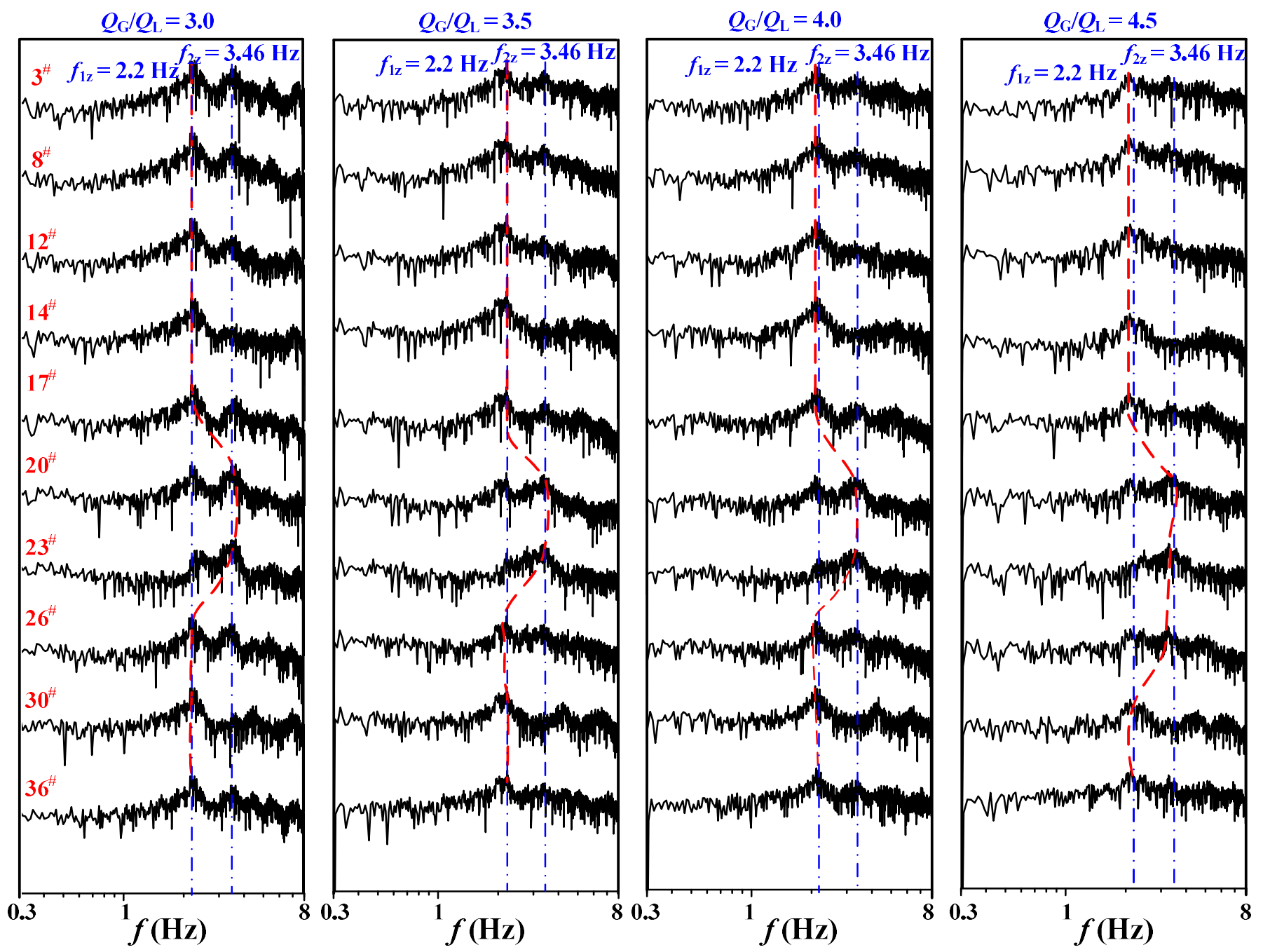

Fig. 15. (continued). 


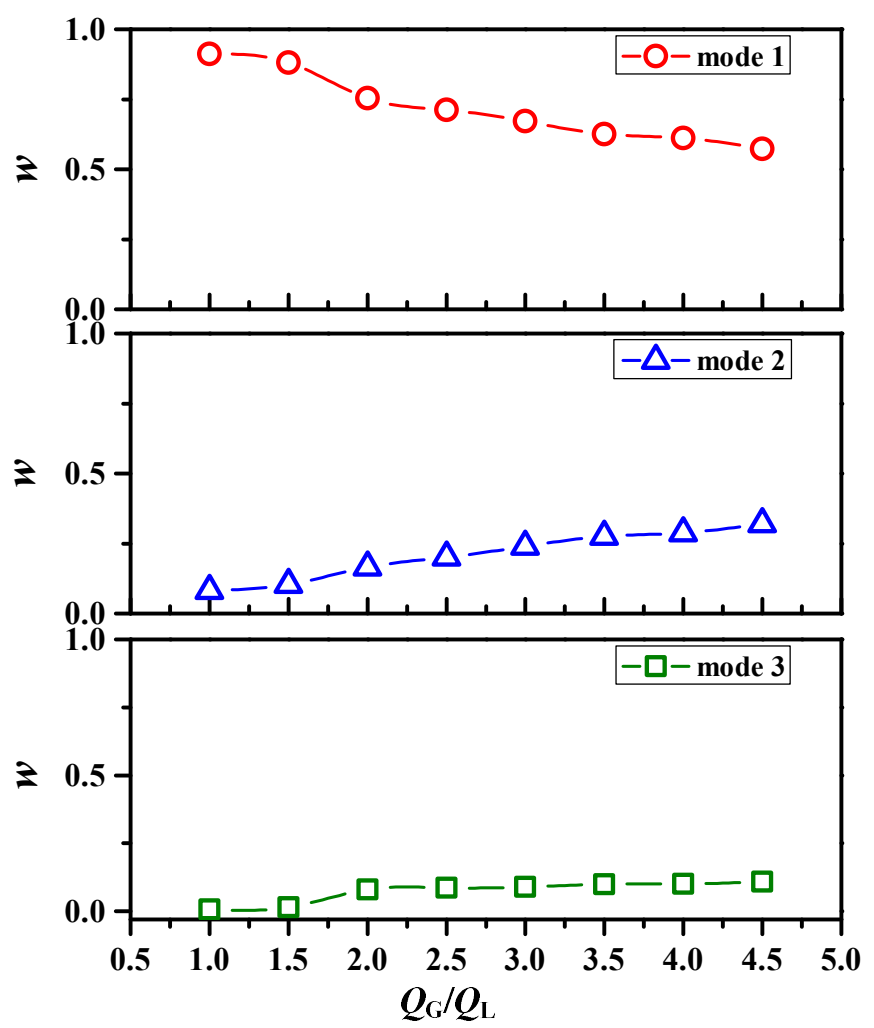

Fig. 16. Variations of modal weights with the gas-to-liquid flow rate ratios. 


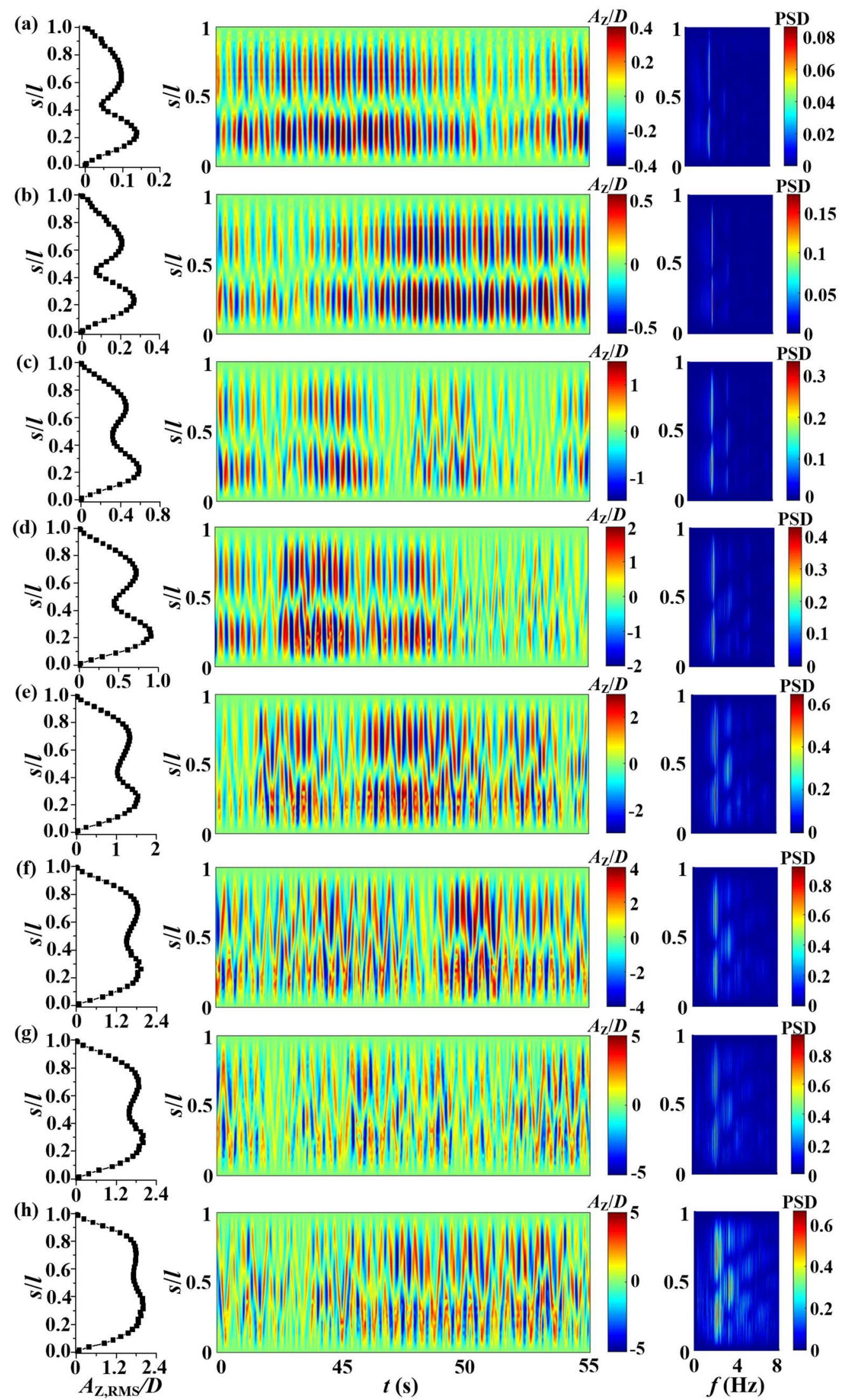

Fig. 17. Riser SIV with RMS amplitude profiles, time-space distributions and PSD amplitudes: (a) $Q_{\mathrm{G}} / Q_{\mathrm{L}}=1.0$; (b) $Q_{\mathrm{G}} / Q_{\mathrm{L}}=1.5$; (c) $Q_{\mathrm{G}} / Q_{\mathrm{L}}=2.0$; (d) $Q_{\mathrm{G}} / Q_{\mathrm{L}}=2.5$; (e) $Q_{\mathrm{G}} / Q_{\mathrm{L}}=3.0$; (f) $Q_{\mathrm{G}} / Q_{\mathrm{L}}=3.5$; (g) $Q_{\mathrm{G}} / Q_{\mathrm{L}}=4.0 ;(\mathrm{h}) Q_{\mathrm{G}} / Q_{\mathrm{L}}=4.5$. 


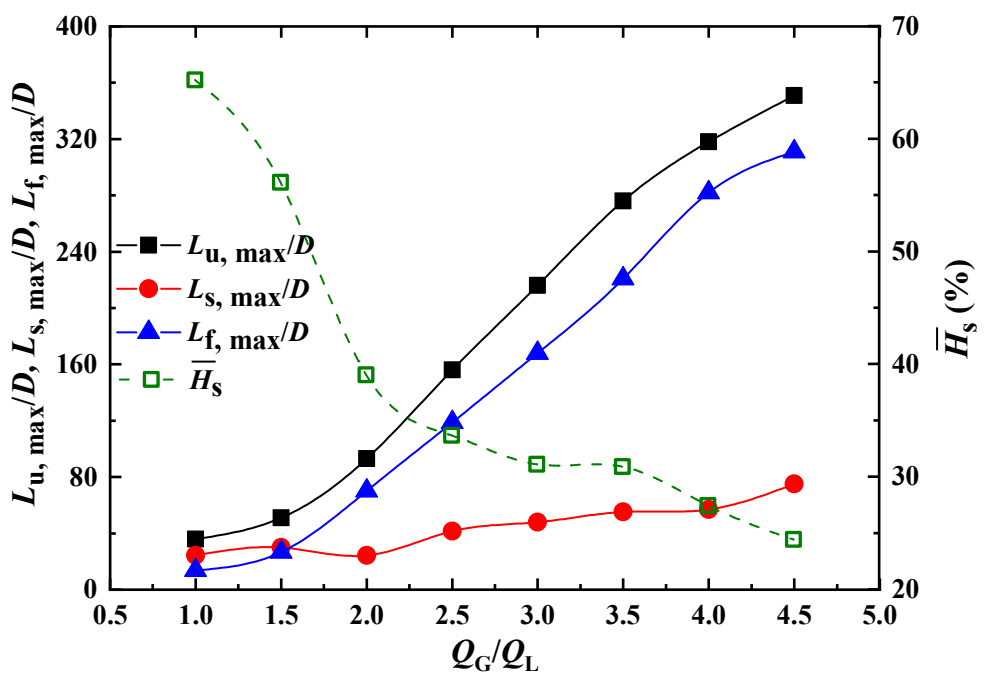

(a)

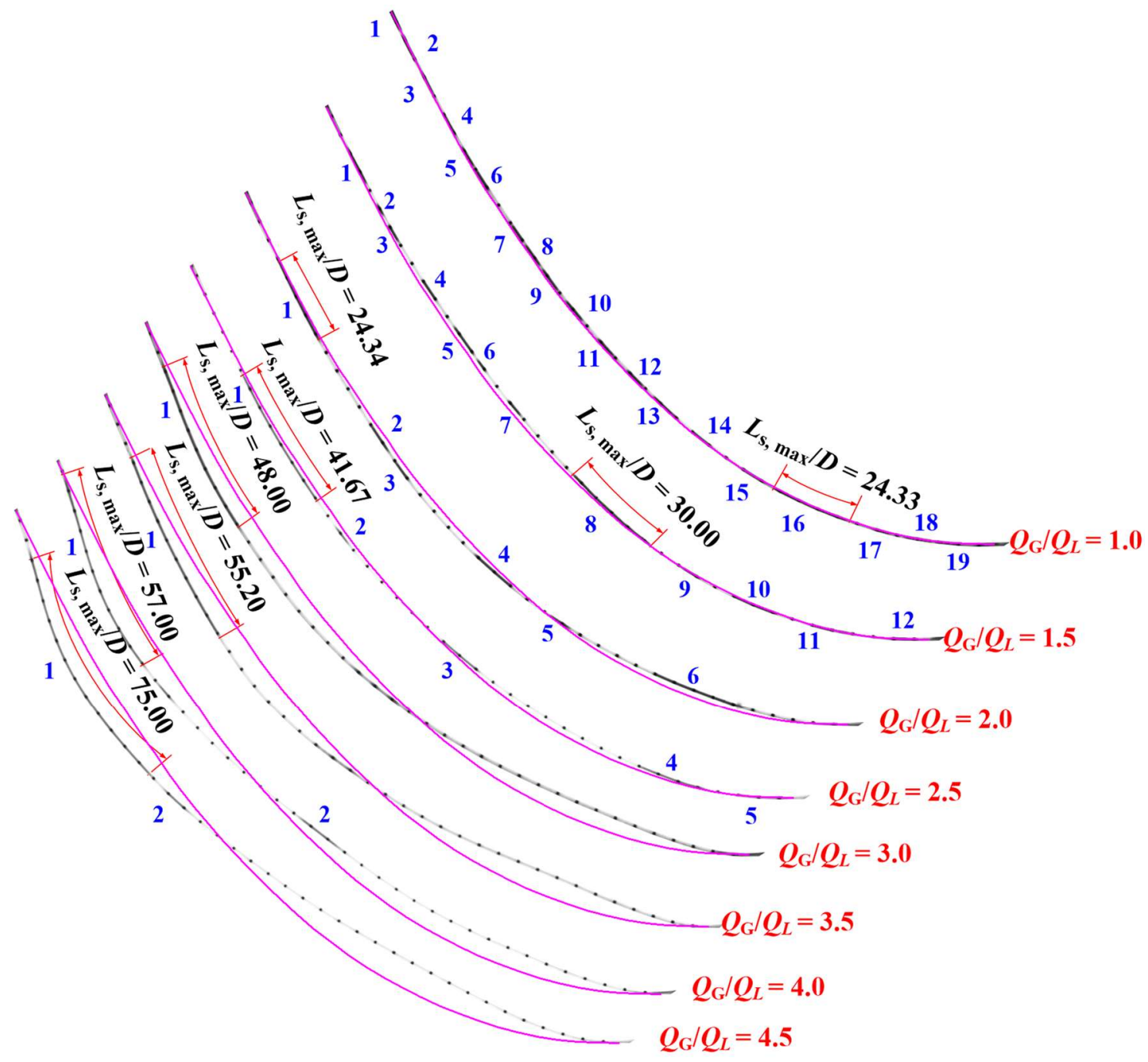

(b)

Fig. 18. Slug flow characteristics at different gas-to-liquid flow rate ratios: (a) variations of slug length and liquid holdup; (b) distributions of liquid inventory along the riser with the longest liquid slug. 

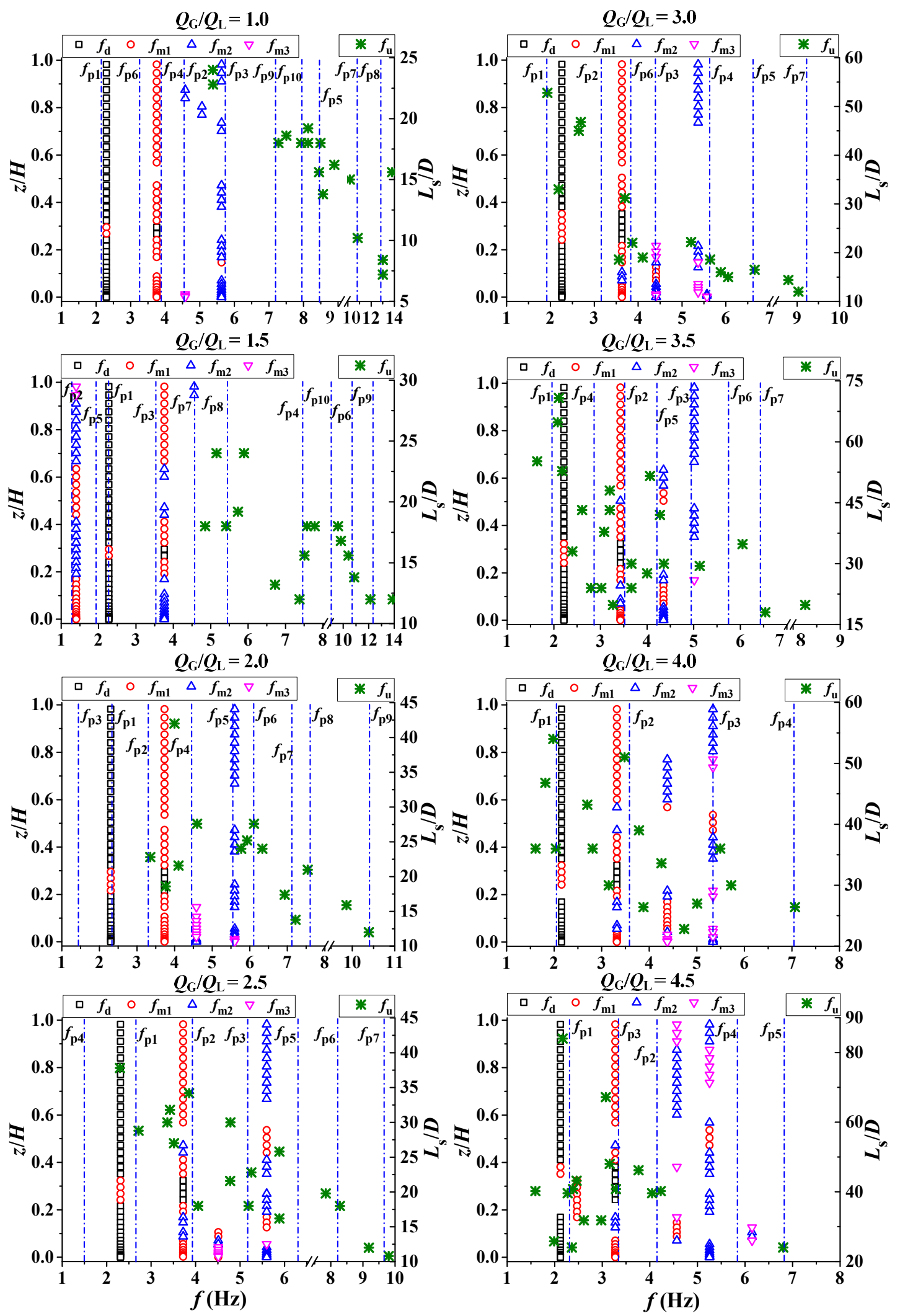

Fig. 19. Comparisons of vibration frequencies, pressure fluctuation frequencies and recurrence frequencies of slugs. 

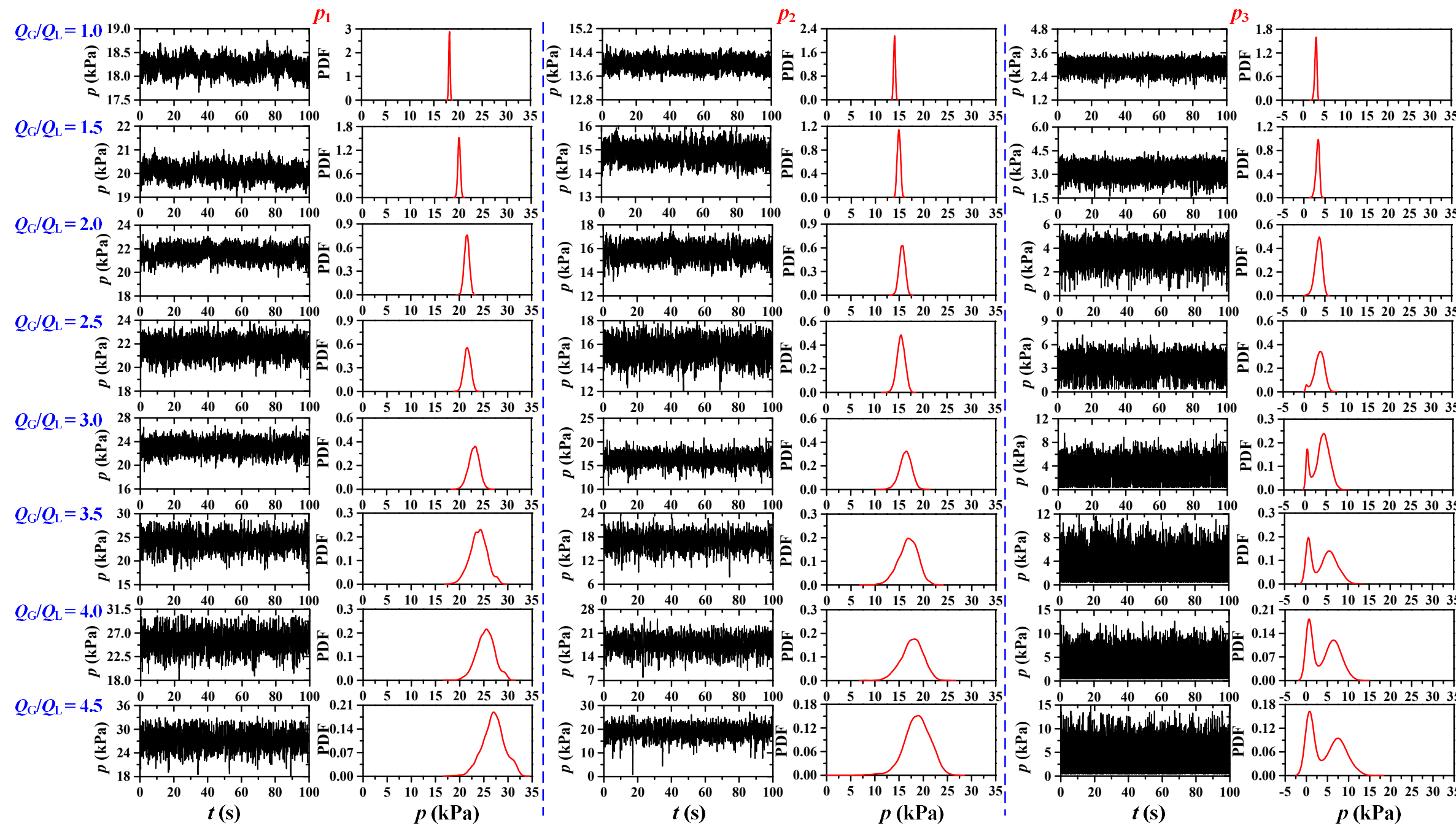

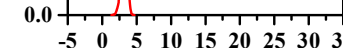

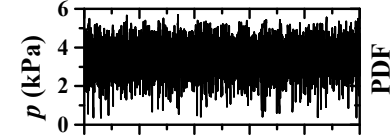

$0.4-1$

$18 \begin{array}{rrrrrr}0 & 20 & 40 & 60 & 80 & 10 \\ 7\end{array}$

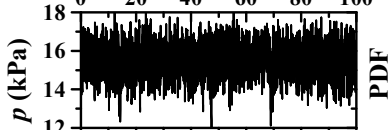

$\begin{array}{llllllllll}0.6 & 0 & 5 & 10 & 15 & 20 & 25 & 30 & 15\end{array}$

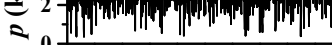

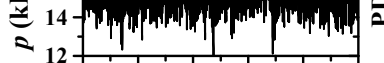
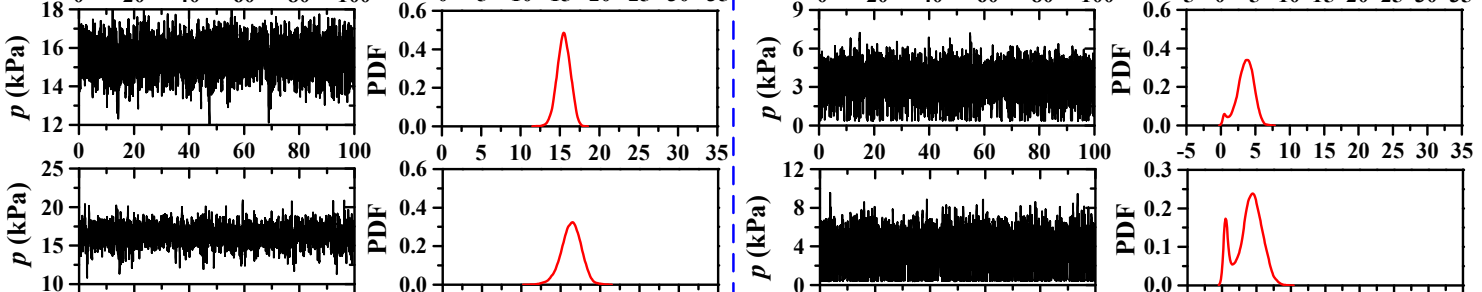

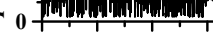
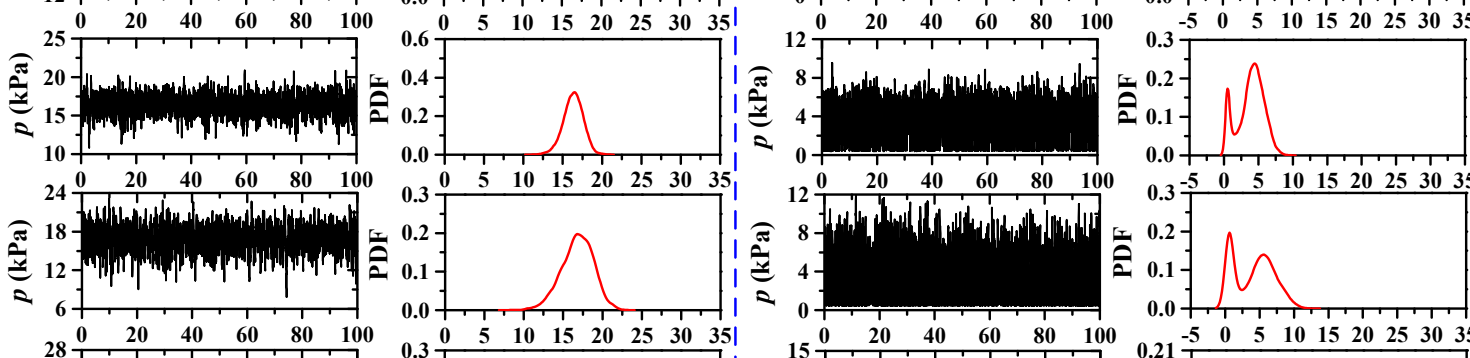

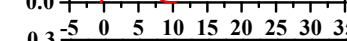

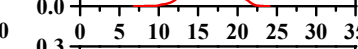
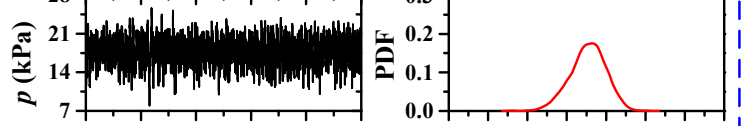

$\begin{array}{llllllllllllllllll}15 & 0 & 20 & 40 & 60 & 80 & 100 & 0.0 & -5 & 0 & 5 & 10 & 15 & 20 & 25 & 30 & 35\end{array}$
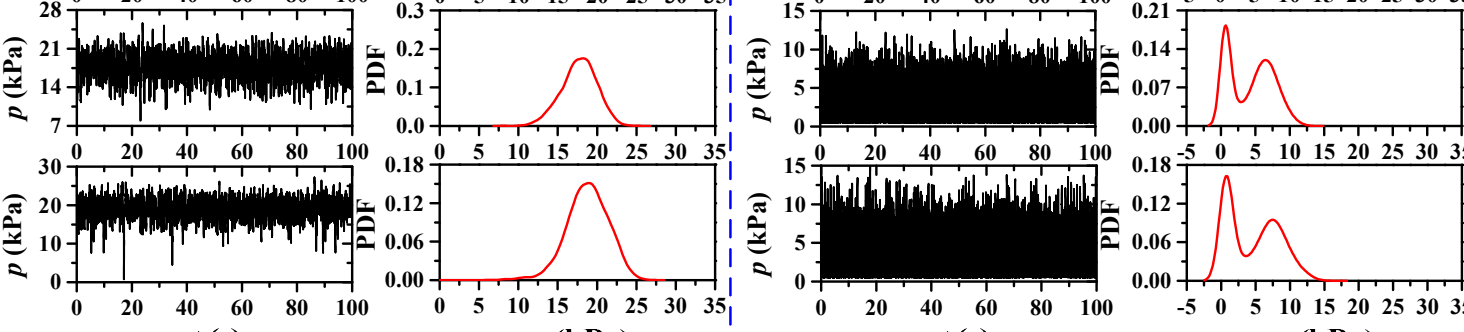

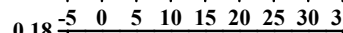

$t(\mathrm{~s})$

$t(\mathrm{~s})$

p (kPa)

$t(\mathbf{s})$

0.

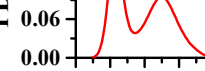

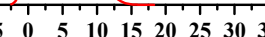

(a) 


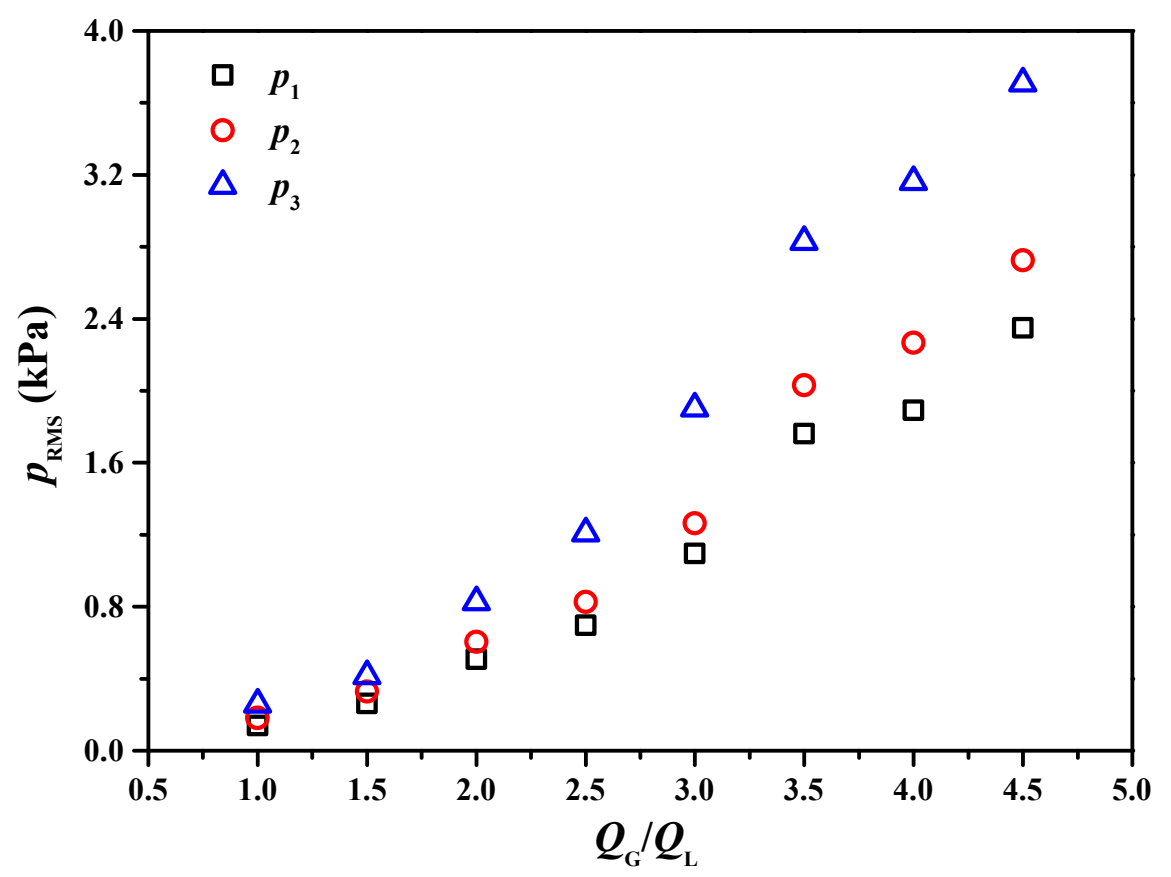

(b)

Fig. 20. Variations of pressure at three monitored points: (a) time histories of pressure and corresponding probability density function; (b) root-mean-squared pressure. 


\section{Highlights}

- Slug flow-induced vibration (SIV) in a catenary riser is experimentally investigated.

- Multi-mode switching in time and standing-travelling wave responses are presented.

- Riser SIV depends on slug length, translational velocity and recurrence frequency.

- Interrelationships between riser dynamics and slug flow characteristics are discussed.

- Riser SIV amplitudes increase with the increased gas-to-liquid flow rate ratio. 